TRANSACTIONS OF THE

AMERICAN MATHEMATICAL SOCIETY

Volume 361, Number 2, February 2009, Pages 1069-1101

S 0002-9947(08)04680-1

Article electronically published on September 29, 2008

\title{
DYNAMICS OF STRONGLY DAMPED WAVE EQUATIONS IN LOCALLY UNIFORM SPACES: ATTRACTORS AND ASYMPTOTIC REGULARITY
}

\author{
MEIHUA YANG AND CHUNYOU SUN
}

\begin{abstract}
This paper is dedicated to analyzing the dynamical behavior of strongly damped wave equations with critical nonlinearity in locally uniform spaces. After proving the global well-posedness, we first establish the asymptotic regularity of the solutions which appears to be optimal and the existence of a bounded (in $H_{l u}^{2}\left(\mathbb{R}^{N}\right) \times H_{l u}^{1}\left(\mathbb{R}^{N}\right)$ ) subset which attracts exponentially every initial $H_{l u}^{1}\left(\mathbb{R}^{N}\right) \times L_{l u}^{2}\left(\mathbb{R}^{N}\right)$-bounded set with respect to the $H_{l u}^{1}\left(\mathbb{R}^{N}\right) \times L_{l u}^{2}\left(\mathbb{R}^{N}\right)$-norm. Then, we show there is a $\left(H_{l u}^{1}\left(\mathbb{R}^{N}\right) \times L_{l u}^{2}\left(\mathbb{R}^{N}\right), H_{\rho}^{1}\left(\mathbb{R}^{N}\right) \times H_{\rho}^{1}\left(\mathbb{R}^{N}\right)\right)$-global attractor, which reflects the strongly damped property of $\Delta u_{t}$ to some extent.
\end{abstract}

\section{INTRODUCTION}

It is well-known that in many cases the long-time behavior of dynamical systems generated by evolution equations of mathematical physics can be described naturally in terms of attractors of corresponding semigroups. In the case where the domain is bounded, the existence of the attractor has been established for a large class of equations; see [3, 9, 21, 24, 32. However, in the case where the domain is unbounded, the situation becomes much more complicated, and we need to confer with more difficulties caused by the unboundedness of the spacial domain. In an unbounded domain typical Sobolev embeddings are not compact and the spaces $L^{p}\left(\mathbb{R}^{N}\right)$ are not nested, which makes our studies much more difficult. Moreover, the usual Sobolev spaces do not include the constant functions and the traveling waves. In order to include these special solutions (e.g., equilibria and relaxation waves) in the attractor, some authors consider bounded and uniformly continuous function spaces and weighted spaces; see [4, 18, 19, 22 and the references therein. Note that weighted spaces ignore the behavior of the solutions for large spatial values and for which the usual Sobolev type embeddings are not available. In 2. 8, 10, 12, 15, 16, 23, 25, 26, 31, 34] etc., the authors apply and develop the properties of locally uniform spaces which enjoy suitable nesting properties, have compact embeddings in weighted spaces and where constant functions lie in them. In these references, the authors study different nonlinear equations and properties

Received by the editors May 18, 2007.

2000 Mathematics Subject Classification. Primary 37L05, 35B40, 35B41.

Key words and phrases. Strongly damped wave equation, locally uniform spaces, critical exponent, asymptotic regularity, attractors.

This work was supported by the NSFC Grants 10601021 and 10726024 and the China Postdoctoral Science Foundation.

(C)2008 American Mathematical Society Reverts to public domain 28 years from publication 
of their nonlinear dynamics in locally uniform spaces, e.g., the authors in [2] systematically study the locally uniform spaces and present a rather complete linear theory of parabolic equations with initial data in locally uniform spaces.

In this paper, we consider the long time behavior of the following semilinear strongly damped wave equation in locally uniform spaces:

$$
u_{t t}+\beta u_{t}-\alpha \Delta u_{t}-\Delta u+f(u)=g, \quad \text { in } \mathbb{R}^{N} \times \mathbb{R}^{+},
$$

with initial data conditions

$$
u(x, 0)=u_{0}(x), \quad u_{t}(x, 0)=v_{0}(x), \quad \text { in } \mathbb{R}^{N},
$$

where $\beta>0, \alpha>0$ and $g \in L_{l u}^{2}\left(\mathbb{R}^{N}\right)$ with $N \geq 3$. The nonlinear term $f$ is a $\mathcal{C}^{1}$-function which satisfies the following conditions:

growth condition

$$
\left|f\left(s_{1}\right)-f\left(s_{2}\right)\right| \leq C\left|s_{1}-s_{2}\right|\left(1+\left|s_{1}\right|^{q}+\left|s_{2}\right|^{q}\right), \quad \forall s_{1}, s_{2} \in \mathbb{R},
$$

where $C$ is a positive constant and $0 \leq q \leq \frac{4}{N-2}$;

dissipation condition

$\exists k \geq 1, \mu_{1}>0, \forall \mu \in\left(0, \mu_{1}\right], \exists C_{\mu} \in \mathbb{R}$,

$$
\begin{aligned}
k F(s)+\mu s^{2}-C_{\mu} \leq s f(s), & \forall s \in \mathbb{R}, \\
\exists c_{0} \in \mathbb{R},-c_{0} \leq F(s), & \forall s \in \mathbb{R},
\end{aligned}
$$

where $F(s)=\int_{0}^{s} f(\tau) d \tau$. These assumptions are the same as that in Cholewa and Dlotko [12].

Following $[2,8,10,12,26,34$, we consider a strictly positive integrable weighted function $\rho: \mathbb{R}^{N} \rightarrow(0, \infty)$ : for $1 \leq p<\infty$, set (see [2, 8, 10, 12, 34, for more details)

$$
L_{\rho}^{p}\left(\mathbb{R}^{N}\right)=\left\{\varphi \in L_{l o c}^{p}\left(\mathbb{R}^{N}\right) ;\|\varphi\|_{L_{\rho}^{p}\left(\mathbb{R}^{N}\right)}=\left(\int_{\mathbb{R}^{N}} \rho(x)|\varphi(x)|^{p} d x\right)^{\frac{1}{p}}<\infty\right\}
$$

let $\tau_{y} \rho(x)=\rho_{y}(x)=\rho(x-y), y \in \mathbb{R}^{N}$, and consider the locally uniform spaces

$$
\begin{gathered}
L_{l u}^{p}\left(\mathbb{R}^{N}\right)=\left\{\varphi \in L_{l o c}^{p}\left(\mathbb{R}^{N}\right) ;\|\varphi\|_{L_{l u}^{p}\left(\mathbb{R}^{N}\right)}=\sup _{y \in \mathbb{R}^{N}}\|\varphi\|_{L_{\rho_{y}}^{p}\left(\mathbb{R}^{N}\right)}<\infty\right\}, \\
\dot{L}_{l u}^{p}\left(\mathbb{R}^{N}\right)=\left\{\varphi \in L_{l u}^{p}\left(\mathbb{R}^{N}\right) ;\left\|\tau_{y} \varphi-\varphi\right\|_{L_{l u}^{p}\left(\mathbb{R}^{N}\right)} \rightarrow 0 \text { as }|y| \rightarrow 0\right\},
\end{gathered}
$$

where $\dot{L}_{l u}^{p}\left(\mathbb{R}^{N}\right)$ is the closed subspace of $L_{l u}^{p}\left(\mathbb{R}^{N}\right)$ consisting of all its elements that are translation continuous; see [2]. The locally uniform Sobolev spaces $W_{l u}^{m, p}\left(\mathbb{R}^{N}\right)$ and $\dot{W}_{l u}^{m, p}\left(\mathbb{R}^{N}\right)$ are defined, respectively, by $L_{l u}^{p}\left(\mathbb{R}^{N}\right)$ and $\dot{L}_{l u}^{p}\left(\mathbb{R}^{N}\right)$ in a way similar to the standard $W^{m, p}\left(\mathbb{R}^{N}\right)$.

We consider strictly positive integrable weighted functions $\rho \in \mathcal{C}^{2}\left(\mathbb{R}^{N}\right)$ satisfying

$$
\left|\frac{\partial \rho}{\partial x_{j}}(x)\right| \leq \rho_{0} \rho(x), \quad\left|\frac{\partial^{2} \rho}{\partial x_{j} \partial x_{k}}(x)\right| \leq \rho_{1} \rho(x), \quad x \in \mathbb{R}^{N}, j, k=1, \cdots, N
$$


with certain positive constants $\rho_{0}, \rho_{1}$. As shown in 2, 8, 10, 12, arbitrary integrable strictly positive functions $\rho \in \mathcal{C}^{2}\left(\mathbb{R}^{N}\right)$ satisfying (1.6) will lead to the same locally uniform spaces $W_{l u}^{k, p}\left(\mathbb{R}^{N}\right), k=0,1,2, p \geq 1$, up to equivalent norms. Hence, in this paper, we consider the exemplary weighted functions

$$
\rho(x)=\left(1+\eta|x|^{2}\right)^{-l}, \text { with } l>\frac{N}{2}, \eta>0,
$$

where, obviously, one can obtain the estimates that $|\nabla \rho| \leq C \sqrt{\eta} \rho$ and $|\Delta \rho| \leq C \eta \rho$.

Problem (1.1)-(1.2) has many relevant physical applications (e.g., see [28] for a summary), and its dynamics have been studied extensively by many authors; see [5, 6, 7, 13, 27, 28, and the reference therein. The existence and smoothness of the global attractor for the case where the spacial domain is bounded have been achieved recently, e.g., see [6, 7, 27, 28. The existence of an $\left(H^{1}\left(\mathbb{R}^{3}\right) \times L^{2}\left(\mathbb{R}^{3}\right), H^{1}\left(\mathbb{R}^{3}\right) \times L^{2}\left(\mathbb{R}^{3}\right)\right)$-global attractor has been proved in Belleri and Pata [5], and further improved results are presented by Conti et al. in [13]. Furthermore, when the spacial domain is a bounded domain $\Omega$, for the system (1.1)-(1.2) with homogeneous Dirichlet boundary conditions, the authors in [27] and [28 have proven that the corresponding $\left(H_{0}^{1}(\Omega) \times L^{2}(\Omega), H_{0}^{1}(\Omega) \times L^{2}(\Omega)\right)$-global attractor $\mathcal{A}_{\mathcal{H}}$ is in fact bounded in $H^{2}(\Omega) \times H_{0}^{1}(\Omega)$ for subcritical and critical cases respectively.

Under the framework of locally uniform spaces, the authors in [10, 16, 31, 34] have considered the weak dissipative wave equations, i.e., $\alpha=0$ and $\beta>0$.

About system (1.1)-(1.2), more recently, under the assumptions of (1.3)-(1.5), Cholewa and Dlotko 12 have obtained the global well-posedness results in the locally uniform spaces $\dot{W}_{l u}^{2, p}\left(\mathbb{R}^{N}\right) \times \dot{L}_{l u}^{p}\left(\mathbb{R}^{N}\right)$ with $p>\frac{N}{2}, p \geq 2$, and proved that their solutions generated a $\mathcal{C}^{0}$ semigroup $\{S(t)\}_{t \geq 0}$ in this space. Furthermore, they also have considered the existence of the global attractor for $\{S(t)\}_{t \geq 0}$. They proved that $\{S(t)\}_{t \geq 0}$ has a bounded $\left(\dot{W}_{l u}^{2, p}\left(\mathbb{R}^{N}\right) \times \dot{L}_{l u}^{p}\left(\mathbb{R}^{N}\right), \dot{W}_{l u}^{2, p}\left(\mathbb{R}^{N}\right) \times \dot{W}_{l u}^{2, p}\left(\mathbb{R}^{N}\right)\right)$-absorbing set, but for the attractor, they only obtained the $\left(\dot{W}_{l u}^{2, p}\left(\mathbb{R}^{N}\right) \times \dot{L}_{l u}^{p}\left(\mathbb{R}^{N}\right), W_{\rho}^{1, p}\left(\mathbb{R}^{N}\right) \times W_{\rho}^{1, p}\left(\mathbb{R}^{N}\right)\right)$-global attractor for the subcritical case, i.e., $q<\frac{4}{N-2}$. The number $q=\frac{4}{N-2}$ is called a critical exponent, since the nonlinearity $f$ is not compact in this case (i.e., for a bounded subset $B \subset \dot{H}_{l u}^{1}\left(\mathbb{R}^{N}\right)$, in general, $f(B)$ is not precompact in $L_{\rho}^{\frac{2 N}{N+2}}\left(\mathbb{R}^{N}\right)$ ). This is an essential difficulty in studying the asymptotic behavior, even for the case that the spacial domain is bounded, e.g., see [6, 27, 28.

The main aim of this paper is to give a detailed analysis of the long-time behavior for the solutions of (1.1)-(1.5) in locally uniform spaces $W_{l u}^{1,2}\left(\mathbb{R}^{N}\right) \times L_{l u}^{2}\left(\mathbb{R}^{N}\right)$, including the global well-posedness and asymptotic regularity of solutions, as well as some exponential attraction. Moreover, we try to show (or highlight) the strongly damped property of $\Delta u_{t}$. For example, we prove a slightly stronger attraction, the $\left(W_{l u}^{1,2}\left(\mathbb{R}^{N}\right) \times L_{l u}^{2}\left(\mathbb{R}^{N}\right), W_{\rho}^{1,2}\left(\mathbb{R}^{N}\right) \times W_{\rho}^{1,2}\left(\mathbb{R}^{N}\right)\right)$-attractor. In other words, for the second ingredient $u_{t}(t)$ of the solution $\left(u(t), u_{t}(t)\right)$, it should behave like the solution of a parabolic equation, which will reflect the strongly damped properties of $\Delta u_{t}$ to some extent.

This paper is organized as follows.

In section 2, we first recall some function spaces and list some short notation which will be used throughout this paper; then we summarize some technical propositions which are useful for our latter proofs and may also be interesting to other people who work in locally uniform spaces. 
In sections 3-5, we consider the global well-posedness of problem (1.1)-(1.2) in $W_{l u}^{1,2}\left(\mathbb{R}^{N}\right) \times L_{l u}^{2}\left(\mathbb{R}^{N}\right)$. Using the methods/framework of [11, 12, and combining some techniques in [31, 34] which are used to obtain some continuities, we define the global weak solutions (see Definition 5.1) by approximations, which generate a continuous semigroup in $W_{l u}^{1,2}\left(\mathbb{R}^{N}\right) \times L_{l u}^{2}\left(\mathbb{R}^{N}\right)$; see Theorems 3.2 and 5.4 .

Then, after some preliminary a priori estimates given in sections 6-8, by using the methods in [35] we overcome the difficulty caused by the critical nonlinearity, and prove the following asymptotic regularity result in section 9, which appears to be optimal:

Theorem 1.1 (Asymptotic regularity). Let $f$ satisfy (1.3)-(1.5), $g \in L_{l u}^{2}\left(\mathbb{R}^{N}\right)$, and $\{S(t)\}_{t \geq 0}$ be the semigroup generated by the global weak solutions of (1.1)(1.2) with the initial data $\left(u_{0}, v_{0}\right) \in W_{l u}^{1,2}\left(\mathbb{R}^{N}\right) \times L_{l u}^{2}\left(\mathbb{R}^{N}\right)$. Then, there exist a set $\mathcal{B}_{1} \subset W_{l u}^{2,2}\left(\mathbb{R}^{N}\right) \times W_{l u}^{1,2}\left(\mathbb{R}^{N}\right)$ (closed and bounded in $W_{\text {lu }}^{2,2} \times W_{\text {lu }}^{1,2}$ ), a positive constant $\nu$ and a monotonically increasing function $Q(\cdot)$ such that: for any bounded (in $W_{l u}^{1,2} \times L_{l u}^{2}$ ) set $B \subset W_{l u}^{1,2}\left(\mathbb{R}^{N}\right) \times L_{l u}^{2}\left(\mathbb{R}^{N}\right)$, the following estimate holds:

$$
\operatorname{dist}_{W_{l u}^{1,2} \times L_{l u}^{2}}\left(S(t) B, \mathcal{B}_{1}\right) \leq Q\left(\|B\|_{W_{l u}^{1,2} \times L_{l u}^{2}}\right) e^{-\nu t},
$$

where dist $_{W_{l u}^{1,2} \times L_{l u}^{2}}$ denotes the usual Hausdorff semidistance in $W_{l u}^{1,2}\left(\mathbb{R}^{N}\right) \times$ $L_{l u}^{2}\left(\mathbb{R}^{N}\right)$.

This theorem says that asymptotically, the solutions are exponentially close or approach more regular solutions in a slightly stronger norm: the $W_{l u}^{1,2} \times L_{l u}^{2}$-norm instead of $W_{\rho}^{1,2} \times L_{\rho}^{2}$-norm, although the global attractor $\mathcal{A}$ obtained in Theorem 1.2 below attracts them only in a weighted space topology.

It is worth noting that Theorem 1.1 is also interesting in the bounded domain case (e.g., see [27, 29, 30]), and to the authors' knowledge, this is the first regularity result in locally uniform space for the wave-type equations with critical nonlinearity. Moreover, it is also a basis for further considering the asymptotic behavior. For example, based on this result, we are constructing (in [33]) a infinitedimensional exponential attractor for (1.1) (see [14] for the corresponding results of parabolic equations); simultaneously, combining some continuity with respect to the $W_{\rho}^{1,2}\left(\mathbb{R}^{N}\right) \times W_{\rho}^{1,2}\left(\mathbb{R}^{N}\right)$-topology, we have the following existence result about the attractor:

Theorem 1.2 (Global attractors). Assume $f$ satisfies (1.3)-(1.5) and $g \in$ $L_{\text {lu }}^{2}\left(\mathbb{R}^{N}\right)$. Then the semigroup $\{S(t)\}_{t \geq 0}$ generated by the global weak solutions of (1.1)-(1.2) with the initial data $\left(u_{0}, v_{0}\right) \in W_{l u}^{1,2}\left(\mathbb{R}^{N}\right) \times L_{l u}^{2}\left(\mathbb{R}^{N}\right)$ has a unique $\left(W_{l u}^{1,2}\left(\mathbb{R}^{N}\right) \times L_{l u}^{2}\left(\mathbb{R}^{N}\right), W_{\rho}^{1,2}\left(\mathbb{R}^{N}\right) \times W_{\rho}^{1,2}\left(\mathbb{R}^{N}\right)\right)$-global attractor $\mathcal{A}$, which satisfies

(i) $\mathcal{A}$ is compact in $W_{\rho}^{1,2}\left(\mathbb{R}^{N}\right) \times W_{\rho}^{1,2}\left(\mathbb{R}^{N}\right)$ and attracts every bounded subset of $W_{\text {lu }}^{1,2}\left(\mathbb{R}^{N}\right) \times L_{\text {lu }}^{2}\left(\mathbb{R}^{N}\right)$ with respect to the $W_{\rho}^{1,2}\left(\mathbb{R}^{N}\right) \times W_{\rho}^{1,2}\left(\mathbb{R}^{N}\right)$-norm;

(ii) $\mathcal{A}$ is closed and bounded in $W_{l u}^{2,2}\left(\mathbb{R}^{N}\right) \times W_{l u}^{1,2}\left(\mathbb{R}^{N}\right)$;

(iii) $\mathcal{A}$ is invariant; that is, $S(t) \mathcal{A}=\mathcal{A}$ for any $t \geq 0$.

For convenience, hereafter let $\|\cdot\|_{\dot{W}_{l u}^{m, p}},\|\cdot\|_{W_{l u}^{m, p}},\|\cdot\|_{W_{\rho}^{m, p}}$ and $\|\cdot\|_{W^{m, p}}$ be the norms of $\dot{W}_{l u}^{m, p}\left(\mathbb{R}^{N}\right), W_{l u}^{m, p}\left(\mathbb{R}^{N}\right), W_{\rho}^{m, p}\left(\mathbb{R}^{N}\right)$ and $W^{m, p}\left(\mathbb{R}^{N}\right)$, respectively. Also, let $\langle\cdot, \cdot\rangle$ be the usual inner product in $L^{2}\left(\mathbb{R}^{N}\right)$ and let $C$ be an arbitrary positive constant, which may be different from line to line or even in the same line. 
Here and later on we omit the domain $\mathbb{R}^{N}$ when the function space is defined over $\mathbb{R}^{N}$, e.g., write $W_{l u}^{m, p}\left(\mathbb{R}^{N}\right)$ as $W_{l u}^{m, p}$; otherwise we will specifically point it out.

\section{Preliminaries}

First, we recall the uniform space $W_{U}^{s, p}\left(\mathbb{R}^{N}\right), s \in \mathbb{R}^{+} \cup\{0\}$ (see [2, 15, 16, 26]), the Banach space consisting of all $\phi \in W_{l o c}^{s, p}\left(\mathbb{R}^{N}\right)$ such that

$$
\|\phi\|_{W_{U}^{s, p}\left(\mathbb{R}^{N}\right)}=\sup _{y \in \mathbb{R}^{N}}\|\phi\|_{W^{s, p}(B(y, 1))}<\infty
$$

where $B(y, 1)=\left\{x \in \mathbb{R}^{N}|| x-y \mid \leq 1\right\}$.

From [2], we know that, for the weighted function $\rho$ satisfying (1.6) in $L_{l u}^{p}$, the following two norms are equivalent: there exist $C_{1}, C_{2}$ such that for all $u \in L_{l u}^{p}$,

$$
\|u\|_{L_{l u}^{p}}^{p}=\sup _{y \in \mathbb{R}^{N}} \int_{\mathbb{R}^{N}} \rho(x-y)|u(x)|^{p} d x \leq C_{1} \sup _{y \in \mathbb{R}^{N}} \int_{B(y, 1)}|u(x)|^{p} d x \leq C_{2}\|u\|_{L_{l u}^{p}}^{p} .
$$

Notice that inequality (2.1) implies that for $k \in \mathbb{N} \cup\{0\}$, uniform space $W_{U}^{k, p}$ and locally uniform space $W_{l u}^{k, p}$ coincide algebraically and topologically when the weighted function $\rho$ satisfies (1.6). Furthermore, by intermediate spaces we know that the same holds for $W_{U}^{s, p}$ and $W_{l u}^{s, p}$ with $s \in \mathbb{R}^{+} \cup\{0\}$ (see Proposition 4.1 of [2]), and we will use this equivalence frequently in our paper.

Notation. For $s \in \mathbb{R}$, we denote $H_{U}^{s}=W_{U}^{s, 2}$ and

$$
\mathcal{H}_{s}=H_{U}^{s+1} \times H_{U}^{s}=W_{U}^{(s+1), 2} \times W_{U}^{s, 2}
$$

with the norm

$$
\|(u, v)\|_{\mathcal{H}_{s}}^{2}=\|u\|_{W_{U}^{(s+1), 2}}^{2}+\|v\|_{W_{U}^{s, 2}}^{2}
$$

Then, in particular,

$$
\begin{gathered}
\mathcal{H}_{0}=W_{U}^{1,2} \times L_{U}^{2}=H_{U}^{1} \times L_{U}^{2}=H_{l u}^{1} \times L_{l u}^{2}, \\
\mathcal{H}_{1}=W_{U}^{2,2} \times W_{U}^{1,2}=H_{U}^{2} \times H_{U}^{1}=H_{l u}^{2} \times H_{l u}^{1} .
\end{gathered}
$$

For the latter applications, we next recall some properties of locally uniform spaces.

Lemma $2.1([2])$. (i) If $s_{1} \geq s_{2} \geq 0,1<p_{1} \leq p_{2}<\infty$ and $s_{1}-\frac{N}{p_{1}} \geq s_{2}-\frac{N}{p_{2}}$, then

$$
W_{U}^{s_{1}, p_{1}}\left(\mathbb{R}^{N}\right) \hookrightarrow W_{U}^{s_{2}, p_{2}}\left(\mathbb{R}^{N}\right)
$$

is continuous. Moreover,

$$
\|\phi\|_{W_{U}^{s, p}} \leq C\|\phi\|_{W_{U}^{s_{1}, p_{1}}}^{\theta}\|\phi\|_{W_{U}^{s_{2}, p_{2}}}^{1-\theta},
$$

where $\theta \in[0,1], \frac{1}{p} \leq \frac{\theta}{p_{1}}+\frac{1-\theta}{p_{2}}, 1<p, p_{1}, p_{2}<\infty$ and

$$
s-\frac{N}{p} \leq \theta\left(s_{1}-\frac{N}{p_{1}}\right)+(1-\theta)\left(s_{2}-\frac{N}{p_{2}}\right) .
$$


(ii) If $\rho$ satisfies (1.6), then the inclusion $W_{U}^{s_{1}, p_{1}}\left(\mathbb{R}^{N}\right) \hookrightarrow W_{\rho}^{s_{2}}, p_{2}\left(\mathbb{R}^{N}\right)$ is compact provided that $s_{2} \in \mathbb{N}, s_{1}>s_{2}, 1<p_{1} \leq p_{2}<\infty$ and $s_{1}-\frac{N}{p_{1}}>s_{2}-\frac{N}{p_{2}}$.

Lemma $2.2([2])$. Let $\alpha \in[0,1]$. Then there exist positive constants $C_{1, \alpha}$ and $C_{2, \alpha}$ such that

$$
C_{1, \alpha}\|\phi\|_{W_{U}^{2 \alpha, 2}}^{2} \leq\|\phi\|_{L_{U}^{2}}^{2}+\left\|(-\Delta)^{\alpha} \phi\right\|_{L_{U}^{2}}^{2} \leq C_{2, \alpha}\|\phi\|_{W_{U}^{2 \alpha, 2}}^{2}
$$

for all $\phi \in W_{U}^{2 \alpha, 2}$.

This lemma provides the theoretical feasibility for us to use fractional power operators, consequently, the bootstrap methods; see $\S 7-\S 8$.

On the other hand, from the definition of $\rho$ (in (1.7) ), obviously, we have

Proposition 2.3. There exist $C_{1}, C_{2}$ such that

$$
C_{1} \rho(x) \leq \inf _{y \in B(x, 1)} \rho(y) \leq \sup _{y \in B(x, 1)} \rho(y) \leq C_{2} \rho(x) \quad \text { for all } x \in \mathbb{R}^{N},
$$

where $B(x, 1)=\left\{y \in \mathbb{R}^{N}|| y-x \mid \leq 1\right\}$.

Then, similar to Proposition 1.2 of 34], we have the following property

Proposition 2.4. There exist $C_{1}, C_{2}$ such that for all $u \in L_{\rho}^{p}(1 \leq p<\infty)$,

$$
C_{1} \int_{\mathbb{R}^{N}} \rho(x)|u(x)|^{p} d x \leq \int_{\mathbb{R}^{N}} \rho(y) \int_{B(y, 1)}|u(x)|^{p} d x d y \leq C_{2} \int_{\mathbb{R}^{N}} \rho(x)|u(x)|^{p} d x .
$$

Combining Proposition 2.4 with the interpolation inequality for bounded domain, we can get the following interpolation inequalities in weighted spaces and locally uniform spaces:

Proposition 2.5. For any $p \in\left[2, \frac{2 N}{N-2}\right]$ and $\theta \in[0,1]$, we have

$$
\|\varphi\|_{L_{\rho}^{p}} \leq C\|\varphi\|_{H_{l u}^{1}}^{\theta}\|\varphi\|_{L_{\rho}^{r}}^{1-\theta} \quad \text { and }\|\varphi\|_{L_{\rho}^{p}} \leq C\|\varphi\|_{H_{\rho}^{1}}^{\theta}\|\varphi\|_{L_{l u}^{r}}^{1-\theta},
$$

where $\frac{1}{p} \leq \frac{\theta}{2}+\frac{1-\theta}{r}$ and $-\frac{N}{p} \leq \theta\left(1-\frac{N}{2}\right)-(1-\theta) \frac{N}{r}$.

Proof. We only give a proof for the first inequality; the second can be obtained by the same method:

$$
\begin{aligned}
\int_{\mathbb{R}^{N}} & \rho(x)|\varphi(x)|^{p} d x \leq C \int_{\mathbb{R}^{N}} \rho(y) \int_{B(y, 1)}|\varphi(x)|^{p} d x d y \\
& \leq C \int_{\mathbb{R}^{N}} \rho(y)\|\varphi\|_{H^{1}(B(y, 1))}^{p \theta}\|\varphi\|_{L^{r}(B(y, 1))}^{p(1-\theta)} d y \\
& \leq C \sup _{y \in \mathbb{R}^{N}}\|\varphi\|_{H^{1}(B(y, 1))}^{p \theta} \int_{\mathbb{R}^{N}} \rho(y)\|\varphi\|_{L^{r}(B(y, 1))}^{p(1-\theta)} d y \\
& \leq C\|\varphi\|_{H_{l u}^{1}}^{p \theta}\left(\int_{\mathbb{R}^{N}} \rho(y)\|\varphi\|_{L^{r}(B(y, 1))^{r}}^{r} d y\right)^{\frac{p(1-\theta)}{r}}\left(\int_{\mathbb{R}^{N}} \rho(y) d y\right)^{\left(1-\frac{p(1-\theta)}{r}\right)} \\
& \leq C\|\varphi\|_{H_{l u}^{1}}^{p \theta}\left(\int_{\mathbb{R}^{N}} \rho(x)|\varphi(x)|^{r} d x\right)^{\frac{p(1-\theta)}{r}} \\
& \leq C\|\varphi\|_{H_{l u}^{1}}^{p \theta}\|\varphi\|_{L_{\rho}^{r}}^{p(1-\theta)} .
\end{aligned}
$$

Next we give a density lemma, which will be used to define the weak solution. 
Lemma 2.6. Let $\rho(\cdot)$ satisfy (1.6). Then $\dot{H}_{l u}^{1}$ is a dense set of $H_{l u}^{1}$ w.r.t. the $H_{\rho}^{1}$ norm. Moreover, for each $v \in H_{l u}^{1}$, there is a bounded (in $H_{l u}^{1}$ ) sequence $\left\{v_{n}\right\}_{n=1}^{\infty} \subset$ $\dot{H}_{l u}^{1}$ that satisfies $v_{n} \rightarrow v$ w.r.t. the $H_{\rho}^{1}$-norm.

Proof. Let $\theta \in \mathcal{C}^{\infty}$ be a cutoff function: $\theta(s):[0, \infty) \rightarrow[0,1]$,

$$
\theta(s)=1 \text { for } 0 \leq s \leq 1, \quad \theta(s)=0 \text { for } s \geq 2,
$$

and $\left|\theta^{\prime}(s)\right| \leq C_{1}$ for all $s \in[0, \infty)$.

For any $\varepsilon>0$ and each $v \in H_{l u}^{1}$, we know that there exists a positive constant $R=R\left(\varepsilon, \rho,\|v\|_{H_{l u}^{1}}, C_{1}\right)$ such that

$$
\|v\|_{H_{\rho}^{1}\left(\mathbb{R}^{N} \backslash B(0, R)\right)} \leq \frac{\varepsilon}{2 C_{1}} .
$$

Let $\theta_{R}(x)=\theta\left(\frac{|x|^{2}}{R^{2}}\right)$. Then, obviously,

$$
\theta_{R} v \in H_{0}^{1}(B(0,2 R)) \subset \dot{H}_{l u}^{1} \quad \text { and }\left\|\theta_{R} v\right\|_{H_{l u}^{1}}^{2} \leq\left(1+C_{1}\right)\|v\|_{H_{l u}^{1}}^{2} .
$$

Moreover,

$$
\begin{aligned}
& \left\|\theta_{R}(x) v(x)-v(x)\right\|_{H_{\rho}^{1}}^{2} \\
& \leq \int_{\mathbb{R}^{N} \backslash B(0, R)} \rho(x)\left|\left(1-\theta_{R}(x)\right) v(x)\right|^{2}+\rho(x)\left|\nabla\left(\left(1-\theta_{R}(x)\right) v(x)\right)\right|^{2} d x \\
& \leq \int_{\mathbb{R}^{N} \backslash B(0, R)} \rho(x)\left(|v(x)|^{2}+|\nabla v(x)|^{2}\right) d x+C_{\theta_{R}^{\prime}} \int_{\mathbb{R}^{N} \backslash B(0, R)} \rho(x)|v(x)|^{2} d x \\
& \leq \varepsilon .
\end{aligned}
$$

Hence, the conclusion of Lemma 2.6 holds.

Remark 2.7. In fact, let $m, p \in \mathbb{N} \cup\{0\}$ and $\rho(\cdot)$ satisfy (1.6); then $\dot{W}_{l u}^{m, p}$ is dense in $W_{l u}^{m, p}$ w.r.t. the $W_{\rho}^{m, p}$-norm. Moreover, for each $v \in W_{l u}^{m, p}$, there is a bounded (in $W_{l u}^{m, p}$ ) sequence $\left\{v_{n}\right\}_{n=1}^{\infty} \subset \dot{W}_{l u}^{m, p}$ that satisfies $v_{n} \rightarrow v$ w.r.t. the $W_{\rho}^{m, p}$-norm.

\section{The Semigroup Generated By (1.1)-1.2}

In this section, we will consider the well-posedness of system (1.1)-(1.2). To this end, we will use the methods/framework as in 11, 12. In our study, the space $\dot{W}_{l u}^{2, p} \times \dot{L}_{l u}^{p}$ is necessary, where our elliptic operator will enjoy a dense domain. That is, it will generate a strong continuous analytic semigroup.

At first, note that we can rewrite (1.1) as an abstract parabolic equation

$$
\frac{d}{d t}\left(\begin{array}{l}
u \\
v
\end{array}\right)+\left(\begin{array}{cc}
0 & -I \\
-\Delta & \beta I-\alpha \Delta
\end{array}\right)\left(\begin{array}{l}
u \\
v
\end{array}\right)=\left(\begin{array}{c}
0 \\
f(u)+g
\end{array}\right), t>0,
$$

with initial data

$$
\left(\begin{array}{l}
u \\
v
\end{array}\right)_{t=0}=\left(\begin{array}{l}
u_{0} \\
v_{0}
\end{array}\right) \text {. }
$$

From Cholewa and Dlotko 12 we have the following results:

Lemma 3.1 ([12]). For each $\alpha>0$, the strongly damped wave operator

$$
\Pi=\left(\begin{array}{cc}
0 & -I \\
-\Delta & \beta I-\alpha \Delta
\end{array}\right)
$$


with the domain

$$
D(\Pi)=\dot{W}_{l u}^{2, p}\left(\mathbb{R}^{N}\right) \times \dot{W}_{l u}^{2, p}\left(\mathbb{R}^{N}\right)
$$

is a sectorial operator in $\dot{W}_{l u}^{2, p}\left(\mathbb{R}^{N}\right) \times \dot{L}_{l u}^{p}\left(\mathbb{R}^{N}\right)$ for each $p \in(1, \infty)$.

Based on the general result above, we now prove the following global solvability of (1.1)-(1.2). For convenience, without loss of generality we always assume $\alpha=\beta=1$ hereafter.

Theorem 3.2. Assume that $f$ satisfies (1.3)-(1.5) and $g \in \dot{L}_{l u}^{2}$. Then the problem (1.1)-(1.2) defines in $Y=\dot{W}_{l u}^{2,2} \times \dot{L}_{l u}^{2}$ a $\mathcal{C}^{0}$-semigroup $\{\tilde{S}(t)\}_{t \geq 0}$ of global solutions; that is, for each $\left(u_{0}, v_{0}\right) \in Y$ and any $T>0$ there exists a unique solution

$$
\left(u, u_{t}\right) \in \mathcal{C}([0, T], Y) \cap \mathcal{C}^{1}((0, T), Y) \cap \mathcal{C}\left((0, T), \dot{W}_{l u}^{2,2} \times \dot{W}_{l u}^{2,2}\right),
$$

depending continuously upon the initial condition.

Proof. We will complete the proof by two steps.

Step 1. Local well-posedness. This problem can be seen as an abstract semilinear parabolic problem, in $Y$, having the form (3.1)-(3.2). From the standard semigroup theory about local well-posedness (e.g., see [9]) of an abstract parabolic equation, thanks to Lemma 3.1 above, we only need to verify that $(0, f(\cdot)+g)^{T}$, is local Lipschitz in $Y$, which is equivalent to $f(\cdot)$, is local Lipschitz from $\dot{W}_{l u}^{2,2}$ to $\dot{L}_{l u}^{2}$ and will be obtained as follows: For any $u_{1}, u_{2} \in \dot{W}_{l u}^{2,2}$ and any $y \in \mathbb{R}^{N}$, we have

$$
\begin{aligned}
& \int_{\mathbb{R}^{N}} \rho_{y}(x)\left|f\left(u_{1}\right)-f\left(u_{2}\right)\right|^{2} d x \\
& \leq C \int_{\mathbb{R}^{N}} \rho_{y}(x)\left(1+\left|u_{1}\right|^{\frac{4}{N-2}}+\left|u_{2}\right|^{\frac{4}{N-2}}\right)^{2}\left|u_{1}-u_{2}\right|^{2} d x \\
& \leq C \int_{\mathbb{R}^{N}} \rho_{y}(z) \int_{B(z, 1)}\left(1+\left|u_{1}\right|^{\frac{4}{N-2}}+\left|u_{2}\right|^{\frac{4}{N-2}}\right)^{2}\left|u_{1}-u_{2}\right|^{2} d x d z \\
& \leq C^{\prime} \int_{\mathbb{R}^{N}} \rho_{y}(z)\left(\int_{B(z, 1)}\left|u_{1}-u_{2}\right|^{\frac{2 N}{N-4}} d x\right)^{\frac{N-4}{N}} d z \\
& \leq C^{\prime} \int_{\mathbb{R}^{N}} \rho_{y}(z)\left|u_{1}-u_{2}\right|_{W^{2,2}(B(z, 1))}^{2} d z \\
& \leq C^{\prime} \int_{\mathbb{R}^{N}} \rho_{y}\left(\left|\Delta\left(u_{1}-u_{2}\right)\right|^{2}+\left|\nabla\left(u_{1}-u_{2}\right)\right|^{2}+\left|u_{1}-u_{2}\right|^{2}\right) d x .
\end{aligned}
$$

Here we used Proposition 2.4 and the growth condition (1.3), and the constant $C^{\prime}$ here only depends on the constants in Proposition 2.4 and the bounds of $\left\|u_{i}\right\|_{\dot{W}_{l u}^{2,2}}(i=1,2)$.

Thus, the application of a standard perturbation result [9] leads to a local solution to (3.1)-(3.2). That is, there exists $\tau>0$ and a unique $Y$-valued function continuous on $[0, \tau]$ such that the equation

$$
\left(\begin{array}{c}
u(t) \\
v(t)
\end{array}\right)=e^{-\Pi t}\left(\begin{array}{c}
u_{0} \\
v_{0}
\end{array}\right)+\int_{0}^{t} e^{-\Pi(t-s)}\left(\begin{array}{c}
u(s) \\
v(s)
\end{array}\right) d s
$$


is satisfied for $t \in[0, \tau]$. Furthermore, the solution to (3.1)-(3.2) is defined for all $t \geq 0$ unless its $Y$-norm blows up in a finite time.

Step 2. Global existence. To obtain the global existence of solution in $Y$, we need to show its $Y$-norm do not blow up in a finite time.

At first, by the a priori estimates given in Theorem 4.1 (e.g., (4.7)), we know that for each local solution $\left(u(t), u_{t}(t)\right)$ corresponding to initial data $\left(u_{0}, v_{0}\right) \in Y$, its $H_{l u}^{1} \times L_{l u}^{2}$-norm cannot blow up at a finite time.

Then, we only need to show as follows that $\|u(t)\|_{H_{l u}^{2}}$ cannot blow up in a finite time.

Multiplying (1.1) by $-\rho_{y} \Delta u$, we have

$$
\begin{aligned}
& \frac{d}{d t}\left(\frac{1}{2} \int_{\mathbb{R}^{N}} \rho_{y}|\Delta u|^{2}-\int_{\mathbb{R}^{N}} \rho_{y} u_{t} \Delta u\right)+\int_{\mathbb{R}^{N}} \rho_{y}|\Delta u|^{2}-\int_{\mathbb{R}^{N}} \rho_{y} u_{t} \Delta u-\int_{\mathbb{R}^{N}} \rho_{y}\left|\nabla u_{t}\right|^{2} \\
& =\int_{\mathbb{R}^{N}} \rho_{y} f^{\prime}(u)|\nabla u|^{2}+\int_{\mathbb{R}^{N}} \nabla \rho_{y} f(u) \nabla u+\int_{\mathbb{R}^{N}} \rho_{y} g \Delta u+\int_{\mathbb{R}^{N}} \nabla \rho_{y} u_{t} \nabla u_{t},
\end{aligned}
$$

where, similar to (3.3) (or see (4.27) and (4.28)), we have

$$
\begin{gathered}
\left.\left|\int_{\mathbb{R}^{N}} \rho_{y} f^{\prime}(u)\right| \nabla u\right|^{2} \mid \leq C_{\|u\|_{H_{l u}^{1}}} \int_{\mathbb{R}^{N}} \rho_{y}\left(|\Delta u|^{2}+|u|^{2}\right), \\
\left|\int_{\mathbb{R}^{N}} \nabla \rho_{y} f(u) \nabla u\right| \leq C_{\|u\|_{H_{l u}^{1}}}+\frac{1}{4} \int_{\mathbb{R}^{N}} \rho_{y}\left(|\Delta u|^{2}+|u|^{2}\right) .
\end{gathered}
$$

At the same time,

$$
\begin{gathered}
\left|\int_{\mathbb{R}^{N}} \rho_{y} g \Delta u\right| \leq \int_{\mathbb{R}^{N}} \rho_{y}|g|^{2}+\frac{1}{4} \int_{\mathbb{R}^{N}} \rho_{y}|\Delta u|^{2}, \\
\left|\int_{\mathbb{R}^{N}} \nabla \rho_{y} u_{t} \nabla u_{t}\right| \leq \int_{\mathbb{R}^{N}} \rho_{y}\left|u_{t}\right|^{2}+\int_{\mathbb{R}^{N}} \rho_{y}\left|\nabla u_{t}\right|^{2} .
\end{gathered}
$$

Therefore, we have

$$
\frac{d}{d t} E(t) \leq C_{1} E(t)+2 \int_{\mathbb{R}^{N}} \rho_{y}\left|\nabla u_{t}\right|^{2}+C_{2}
$$

where $E(t)=\frac{1}{2} \int_{\mathbb{R}^{N}} \rho_{y}|\Delta u(t)|^{2}-\int_{\mathbb{R}^{N}} \rho_{y} u_{t}(t) \Delta u(t), C_{1}$ depends only on $\|u(t)\|_{H_{l u}^{1}}$, and $C_{2}$ depends only on $\|g\|_{L_{l u}^{2}},\left\|u_{t}(t)\right\|_{L_{l u}^{2}},\|u(t)\|_{H_{l u}^{1}}$. Then from (4.7), we know that $C_{1}, C_{2}$ depend only on $\left(u_{0}, v_{0}\right), g$ and the coefficients in (1.3)-(1.5)).

Hence, applying the Gronwall lemma, we have

$$
E(t) \leq e^{C_{1} t} E(0)+\frac{C_{2}}{C_{1}} e^{C_{1} t}+2 e^{C_{1} t} \int_{0}^{t} \int_{\mathbb{R}^{N}} \rho_{y}\left|\nabla u_{t}(s)\right|^{2} d x d s .
$$

For the last term on the right hand side, integrating (4.5) over $[0, t]$ and using (1.5), we know this term can also be dominated only by $\left(u_{0}, v_{0}\right), g$ and the constant $c_{0}$ in (1.5).

So, by using the Cauchy inequality to (3.4) we get that $\|\Delta u(t)\|_{L_{l u}^{2}}$ cannot blow up at a finite time, which implies the global existence of solutions.

Remark 3.3. From the proof above, we observe (from (3.3) ) that if only for the local well-posedness in $Y$, we can enlarge the growth order $\frac{4}{N-2}$ in (1.3) to $\frac{4}{N-4}$. 
More generally, for a general phase space $\dot{W}_{l u}^{2, p} \times \dot{L}_{l u}^{p}, p \geq 2$ (considered in [12]), if $f$ satisfies the following condition, instead of (1.3),

$$
\left|f\left(s_{1}\right)-f\left(s_{2}\right)\right| \leq C_{0}\left|s_{1}-s_{2}\right|\left(1+\left|s_{1}\right|^{\frac{2 p}{N-2 p}}+\left|s_{2}\right|^{\frac{2 p}{N-2 p}}\right) \quad \forall s_{1}, s_{2} \in \mathbb{R},
$$

then using the same methods as (3.3), we can deduce the following local Lipschitz continuity:

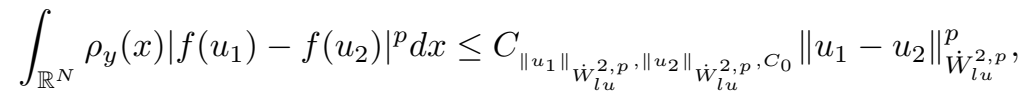

which, combined with Lemma 3.1. immediately implies the local well-posedness results in $\dot{W}_{l u}^{2, p} \times \dot{L}_{l u}^{p}$.

Remark 3.4. Note that in Theorem 3.2 and Remark 3.3. we do not assume that $p$ satisfies the additional assumption $p>\frac{N}{2}$ as required in [12] (to our knowledge, the assumption $p>\frac{N}{2}$ in $\left[12\right.$ is mainly for obtaining the embedding $\dot{W}_{l u}^{2, p}\left(\mathbb{R}^{N}\right) \hookrightarrow$ $L^{\infty}\left(\mathbb{R}^{N}\right)$, which is then used to guarantee the Lipschitz continuity of $f$ ).

\section{A priori estimates for the solutions given in Theorem 3.2}

\subsection{Dissipation estimates in $\mathcal{H}_{0}$.}

Theorem 4.1. Under the assumptions of Theorem 3.2, there is a positive constant $\varrho_{0}$ such that for any bounded (in $\mathcal{H}_{0}$ ) subset $B \subset \dot{W}_{l u}^{2,2} \times \dot{L}_{l u}^{2}$, there exists a positive constant $T_{1}=T_{1}(B)$ which depends only on the $\mathcal{H}_{0}$-bound of $B$ such that

$$
\|u(t)\|_{H_{l u}^{1}}+\left\|u_{t}(t)\right\|_{L_{l u}^{2}} \leq \varrho_{0} \quad \text { for all } t \geq T_{1} \text { and }\left(u_{0}, v_{0}\right) \in B .
$$

Proof. Multiplying (1.1) by $\rho_{y}\left(u_{t}+\frac{1}{2} u\right)$, we have

$$
\begin{aligned}
&\left\langle\left( u_{t}+\right.\right.\left.\left.\frac{1}{2} u\right)_{t}, \rho_{y}\left(u_{t}+\frac{1}{2} u\right)\right\rangle+\frac{1}{2}\left\langle u_{t}, \rho_{y}\left(u_{t}+\frac{1}{2} u\right)\right\rangle-\left\langle\Delta u_{t}, \rho_{y}\left(u_{t}+\frac{1}{2} u\right)\right\rangle \\
&-\left\langle\Delta u, \rho_{y}\left(u_{t}+\frac{1}{2} u\right)\right\rangle+\left\langle f(u), \rho_{y}\left(u_{t}+\frac{1}{2} u\right)\right\rangle=\left\langle g, \rho_{y}\left(u_{t}+\frac{1}{2} u\right)\right\rangle .
\end{aligned}
$$

Next, we deal with each term of (4.1) one by one as follows:

$$
\begin{gathered}
\left\langle\left(u_{t}+\frac{1}{2} u\right)_{t}, \rho_{y}\left(u_{t}+\frac{1}{2} u\right)\right\rangle=\frac{1}{2} \frac{d}{d t} \int_{\mathbb{R}^{N}} \rho_{y}\left|u_{t}+\frac{1}{2} u\right|^{2}, \\
\left\langle\frac{1}{2} u_{t}, \rho_{y}\left(u_{t}+\frac{1}{2} u\right)\right\rangle=\frac{1}{2} \int_{\mathbb{R}^{N}} \rho_{y}\left|u_{t}\right|^{2}+\frac{1}{8} \frac{d}{d t} \int_{\mathbb{R}^{N}} \rho_{y}|u|^{2}, \\
\left\langle-\Delta u_{t}, \rho_{y}\left(u_{t}+\frac{1}{2} u\right)\right\rangle \\
=\int_{\mathbb{R}^{N}} \rho_{y}\left|\nabla u_{t}\right|^{2}+\frac{1}{4} \frac{d}{d t} \int_{\mathbb{R}^{N}} \rho_{y}|\nabla u|^{2}+\int_{\mathbb{R}^{N}} \nabla u_{t} \nabla \rho_{y} u_{t}+\frac{1}{2} \int_{\mathbb{R}^{N}} \nabla u_{t} \nabla \rho_{y} u
\end{gathered}
$$

and

$$
\begin{aligned}
& \left\langle-\Delta u, \rho_{y}\left(u_{t}+\frac{1}{2} u\right)\right\rangle \\
& \quad=\frac{1}{2} \int_{\mathbb{R}^{N}} \rho_{y}|\nabla u|^{2}+\frac{1}{2} \frac{d}{d t} \int_{\mathbb{R}^{N}} \rho_{y}|\nabla u|^{2}+\int_{\mathbb{R}^{N}} \nabla u \nabla \rho_{y} u_{t}+\frac{1}{2} \int_{\mathbb{R}^{N}} \nabla u \nabla \rho_{y} u,
\end{aligned}
$$

where

$$
\left|\int_{\mathbb{R}^{N}} \nabla u_{t} \nabla \rho_{y} u_{t}+\frac{1}{2} \int_{\mathbb{R}^{N}} \nabla u_{t} \nabla \rho_{y} u\right| \leq C \sqrt{\eta} \int_{\mathbb{R}^{N}} \rho_{y}\left(\left|\nabla u_{t}\right|^{2}+\left|u_{t}\right|^{2}+|u|^{2}\right)
$$


and

$$
\left|\int_{\mathbb{R}^{N}} \nabla u \nabla \rho_{y} u_{t}+\frac{1}{2} \int_{\mathbb{R}^{N}} \nabla u \nabla \rho_{y} u\right| \leq C \sqrt{\eta} \int_{\mathbb{R}^{N}} \rho_{y}\left(|\nabla u|^{2}+\left|u_{t}\right|^{2}+|u|^{2}\right) .
$$

On the other hand,

$$
\left\langle f(u), \rho_{y}\left(u_{t}+\frac{1}{2} u\right)\right\rangle=\frac{d}{d t} \int_{\mathbb{R}^{N}} \rho_{y} F(u)+\frac{1}{2} \int_{\mathbb{R}^{N}} \rho_{y} f(u) u
$$

and

$$
\left|\left\langle g, \rho_{y}\left(u_{t}+\frac{1}{2} u\right)\right\rangle\right| \leq \frac{\epsilon}{4} \int_{\mathbb{R}^{N}} \rho_{y}\left(\left|u_{t}\right|^{2}+|u|^{2}\right)+C_{\epsilon} \int_{\mathbb{R}^{N}} \rho_{y}|g|^{2}
$$

Moreover, from (1.4) and (1.5) we have that

$$
k \int_{\mathbb{R}^{N}} \rho_{y} F(u)+\mu \int_{\mathbb{R}^{N}} \rho_{y}|u|^{2}-C_{\mu} \int_{\mathbb{R}^{N}} \rho_{y} \leq \int_{\mathbb{R}^{N}} \rho_{y} f(u) u
$$

and

$$
-c_{0} \int_{\mathbb{R}^{N}} \rho_{y} \leq \int_{\mathbb{R}^{N}} \rho_{y} F(u) .
$$

Substituting the estimates (4.2)-(4.4) into (4.1) simultaneously, choosing $\eta$ and $\epsilon$ small enough, we can obtain that

$$
\begin{aligned}
& \frac{1}{2} \frac{d}{d t} \int_{\mathbb{R}^{N}} \rho_{y} \mid u_{t}+\left.\frac{1}{2} u\right|^{2}+\frac{1}{8} \frac{d}{d t} \int_{\mathbb{R}^{N}} \rho_{y}|u|^{2}+\frac{3}{4} \frac{d}{d t} \int_{\mathbb{R}^{N}} \rho_{y}|\nabla u|^{2}+\frac{d}{d t} \int_{\mathbb{R}^{N}} \rho_{y} F(u) \\
&+\frac{1}{2} \int_{\mathbb{R}^{N}} \rho_{y}\left|\nabla u_{t}\right|^{2}+C_{k, \mu} \int_{\mathbb{R}^{N}} \rho_{y}\left(\left|u_{t}+\frac{1}{2} u\right|^{2}+|u|^{2}+|\nabla u|^{2}+F(u)\right) \\
&(4.5) \quad \leq C_{\epsilon} \int_{\mathbb{R}^{N}} \rho_{y}|g|^{2}+C_{\mu} \int_{\mathbb{R}^{N}} \rho_{y} .
\end{aligned}
$$

Denote

$$
E_{1}(t)=\int_{\mathbb{R}^{N}} \rho_{y}\left(\left|u_{t}(t)+\frac{1}{2} u(t)\right|^{2}+\frac{1}{4}|u(t)|^{2}+\frac{3}{2}|\nabla u(t)|^{2}+2 F(u(t))\right) .
$$

Then, using the Gronwall lemma, we obtain that

$$
E_{1}(t) \leq e^{-C_{k, \mu} t} E_{1}(0)+\frac{C_{\mu, \epsilon}}{C_{k, \mu}} \int_{\mathbb{R}^{N}} \rho_{y}\left(|g|^{2}+1\right) .
$$


Hence, we can conclude Theorem 4.1 immediately with a constant $\varrho_{0}$ which only depends on $\|g\|_{L_{l u}^{2}}, \int_{\mathbb{R}^{N}} \rho(x) d x$ and constants $k, \mu, c_{0}$.

4.2. Strongly damped property. The following results reflect some kind of strongly damped property of $-\Delta u_{t}$ in some sense; see, e.g. [27, 28] for bounded domain cases.

Lemma 4.2. Under the assumptions of Theorem 3.2, there is a positive constant $\varrho_{1}$ such that for any bounded (in $\mathcal{H}_{0}$ ) subset $B \subset \dot{W}_{l u}^{2,2} \times \dot{L}_{l u}^{2}$, there exists a positive constant $T_{2}=T_{2}(B)$ which depends only on the $\mathcal{H}_{0}$-bound of $B$ such that

$$
\|u(t)\|_{H_{l u}^{1}}+\left\|u_{t}(t)\right\|_{H_{l u}^{1}} \leq \varrho_{1} \quad \text { for all } t \geq T_{2} \text { and }\left(u_{0}, v_{0}\right) \in B \text {. }
$$

Proof. Multiplying (1.1) by $\rho_{y}\left(u_{t t}+u_{t}\right)$, we have

$$
\frac{1}{2} \frac{d}{d t} \Lambda\left(\xi_{u}(t)\right)+\Gamma\left(\xi_{u}(t)\right)=0
$$

where

$$
\Lambda\left(\xi_{u}(t)\right)=\int_{\mathbb{R}^{N}} \rho_{y}\left|\nabla u_{t}\right|^{2}+\int_{\mathbb{R}^{N}} \rho_{y}|\nabla u|^{2}+2\left\langle\nabla u, \rho_{y} \nabla u_{t}\right\rangle+2\left\langle f(u), \rho_{y} u_{t}\right\rangle-2\left\langle g, \rho_{y} u_{t}\right\rangle
$$

and

$$
\begin{aligned}
\Gamma\left(\xi_{u}(t)\right)=\int_{\mathbb{R}^{N}} \rho_{y}\left|u_{t t}+u_{t}\right|^{2}-\left\langle f^{\prime}(u) u_{t}, \rho_{y} u_{t}\right\rangle \\
\quad+\left\langle f(u), \rho_{y} u_{t}\right\rangle-\left\langle g, \rho_{y} u_{t}\right\rangle+\left\langle\nabla u_{t}+\nabla u, \nabla \rho_{y}\left(u_{t t}+u_{t}\right)\right\rangle,
\end{aligned}
$$

where $\xi_{u}(t):=\left(u(t), u_{t}(t)\right)$ is the unique solution of (1.1) with initial data $\left(u_{0}, v_{0}\right)$.

Then,

$$
\begin{aligned}
\Gamma\left(\xi_{u}(t)\right) & -\frac{1}{2} \Lambda\left(\xi_{u}(t)\right)=\int_{\mathbb{R}^{N}} \rho_{y}\left|u_{t t}+u_{t}\right|^{2}-\left\langle f^{\prime}(u) u_{t}, \rho_{y} u_{t}\right\rangle-\left\langle\nabla u, \rho_{y} \nabla u_{t}\right\rangle \\
& +\left\langle\nabla u_{t}+\nabla u, \nabla \rho_{y}\left(u_{t t}+u_{t}\right)\right\rangle-\frac{1}{2} \int_{\mathbb{R}^{N}} \rho_{y}\left|\nabla u_{t}\right|^{2}-\frac{1}{2} \int_{\mathbb{R}^{N}} \rho_{y}|\nabla u|^{2} .
\end{aligned}
$$

Note that

$$
\left|2\left\langle\nabla u, \rho_{y} \nabla u_{t}\right\rangle\right| \leq \frac{1}{4} \int_{\mathbb{R}^{N}} \rho_{y}\left|\nabla u_{t}\right|^{2}+4 \int_{\mathbb{R}^{N}} \rho_{y}|\nabla u|^{2},
$$

and using Proposition 2.4, we have

$$
\begin{aligned}
\left|2\left\langle f(u), \rho_{y} u_{t}\right\rangle\right| & \leq C \int_{\mathbb{R}^{N}} \rho_{y}\left(1+|u|+|u|^{\frac{N+2}{N-2}}\right)\left|u_{t}\right| \\
& \leq C \int_{\mathbb{R}^{N}} \rho_{y}(z) \int_{B(z, 1)}\left(1+|u(x)|+|u(x)|^{\frac{N+2}{N-2}}\right)\left|u_{t}(x)\right| d x d z \\
& \leq C \int_{\mathbb{R}^{N}} \rho_{y}(z)\left(1+\|u\|_{H^{1}(B(z, 1))}^{\frac{N+2}{N-2}}\right)\left\|u_{t}\right\|_{H^{1}(B(z, 1))} d z \\
& \leq \frac{1}{4} \int_{\mathbb{R}^{N}} \rho_{y}\left(\left|\nabla u_{t}\right|^{2}+\left|u_{t}\right|^{2}\right)+8 C\left(\int_{\mathbb{R}^{N}} \rho_{y}\left(|\nabla u|^{2}+|u|^{2}+1\right)\right)^{\frac{N+2}{N-2}}
\end{aligned}
$$

and

$$
\left|-2\left\langle g, \rho_{y} u_{t}\right\rangle\right| \leq \frac{1}{4} \int_{\mathbb{R}^{N}} \rho_{y}\left|u_{t}\right|^{2}+4 \int_{\mathbb{R}^{N}} \rho_{y}|g|^{2}
$$


Then, we have that

$$
\begin{aligned}
\Lambda\left(\xi_{u}(t)\right) \geq & \frac{1}{2} \int_{\mathbb{R}^{N}} \rho_{y}\left(\left|\nabla u_{t}\right|^{2}+\left|u_{t}\right|^{2}\right)-\int_{\mathbb{R}^{N}} \rho_{y}\left|u_{t}\right|^{2}-3 \int_{\mathbb{R}^{N}} \rho_{y}|\nabla u|^{2} \\
& -8 C\left(\int_{\mathbb{R}^{N}} \rho_{y}\left(|\nabla u|^{2}+|u|^{2}+1\right)\right)^{\frac{N+2}{N-2}}-4 \int_{\mathbb{R}^{N}} \rho_{y}|g|^{2}
\end{aligned}
$$

and

$$
\begin{aligned}
\Lambda\left(\xi_{u}(t)\right) \leq & 3 \int_{\mathbb{R}^{N}} \rho_{y}\left(\left|\nabla u_{t}\right|^{2}+\left|u_{t}\right|^{2}\right)+5 \int_{\mathbb{R}^{N}} \rho_{y}|\nabla u|^{2} \\
& +8 C\left(\int_{\mathbb{R}^{N}} \rho_{y}\left(|\nabla u|^{2}+|u|^{2}+1\right)\right)^{\frac{N+2}{N-2}}+4 \int_{\mathbb{R}^{N}} \rho_{y}|g|^{2} .
\end{aligned}
$$

Furthermore, applying Proposition 2.4 again, we have

$$
\begin{aligned}
\left|-\left\langle f^{\prime}(u) u_{t}, \rho_{y} u_{t}\right\rangle\right| & \leq C\|u\|_{H_{l u}^{1}}^{\frac{4}{N-2}} \int_{\mathbb{R}^{N}} \rho_{y}\left(\left|\nabla u_{t}\right|^{2}+\left|u_{t}\right|^{2}\right), \\
\left|\left\langle\nabla u, \rho_{y} \nabla u_{t}\right\rangle\right| & \leq \frac{1}{2} \int_{\mathbb{R}^{N}} \rho_{y}\left|\nabla u_{t}\right|^{2}+\frac{1}{2} \int_{\mathbb{R}^{N}} \rho_{y}|\nabla u|^{2}, \\
\left|\left\langle\nabla u_{t}+\nabla u, \nabla \rho_{y}\left(u_{t t}+u_{t}\right)\right\rangle\right| & \leq C \sqrt{\eta} \int_{\mathbb{R}^{N}} \rho_{y}\left(\left|u_{t t}+u_{t}\right|^{2}+\left|\nabla u_{t}\right|^{2}+|\nabla u|^{2}\right) .
\end{aligned}
$$

Summarizing the above estimates into (4.9), and taking $\eta$ small enough, we get that

$$
\begin{aligned}
\Gamma\left(\xi_{u}(t)\right)-\frac{1}{2} \Lambda\left(\xi_{u}(t)\right) \geq \frac{1}{2} \int_{\mathbb{R}^{N}} \rho_{y}\left|u_{t t}+u_{t}\right|^{2} & +\frac{1}{4} \int_{\mathbb{R}^{N}} \rho_{y}\left|\nabla u_{t}\right|^{2}-C \int_{\mathbb{R}^{N}} \rho_{y}\left(|\nabla u|^{2}+|u|^{2}\right) \\
& -C\|u\|_{H_{l u}^{1}}^{\frac{4}{N-2}} \int_{\mathbb{R}^{N}} \rho_{y}\left(\left|\nabla u_{t}\right|^{2}+\left|u_{t}\right|^{2}\right) .
\end{aligned}
$$

Then, substituting the above inequality into (4.8), we obtain that

$$
\begin{aligned}
& \frac{1}{2} \frac{d}{d t} \Lambda\left(\xi_{u}(t)\right)+\frac{1}{2} \Lambda\left(\xi_{u}(t)\right)+\frac{1}{2} \int_{\mathbb{R}^{N}} \rho_{y}\left|u_{t t}+u_{t}\right|^{2} \\
& \quad \leq \int_{\mathbb{R}^{N}} \rho_{y}\left(|\nabla u|^{2}+|u|^{2}\right)+C\|u\|_{H_{l u}^{1}}^{\frac{4}{N-2}} \int_{\mathbb{R}^{N}} \rho_{y}\left(\left|\nabla u_{t}\right|^{2}+\left|u_{t}\right|^{2}\right) .
\end{aligned}
$$

Applying Theorem 4.1. we know that there is a $T_{1}$ which depends on the $\mathcal{H}_{0^{-}}$ bound of $B$ such that

$$
\|u(t)\|_{H_{l u}^{1}}^{2}+\left\|u_{t}(t)\right\|_{L_{l u}^{2}}^{2} \leq \varrho_{0} \text { for all } t \geq T_{1}
$$

therefore, we can rewrite (4.12) as follows: For all $t \geq T_{1}$ and all $\left(u_{0}, v_{0}\right) \in B$, we have

$$
\frac{d}{d t} \Lambda\left(\xi_{u}(t)\right)+\Lambda\left(\xi_{u}(t)\right) \leq C_{1}+C_{2} \int_{\mathbb{R}^{N}} \rho_{y}\left|\nabla u_{t}\right|^{2}
$$

with two proper constants $C_{1}$ and $C_{2}$ which depend only on $\varrho_{0}$ and $\int_{\mathbb{R}^{N}} \rho d x$. Synchronously, following (4.11), we have

$$
\Lambda\left(\xi_{u}(t)\right) \leq 3 \int_{\mathbb{R}^{N}} \rho_{y}\left|\nabla u_{t}(t)\right|^{2}+C_{3} \quad \text { for all } t \geq T_{1} \text { and all }\left(u_{0}, v_{0}\right) \in B
$$

where the constant $C_{3}$ also depends only on $\varrho_{0}, \rho$ and $\|g\|_{L_{l u}^{2}}$. 
On the other hand, we integrate (4.5) over $[t, t+1]$ for any $0 \leq t<\infty$. Then

$$
\begin{aligned}
\int_{t}^{t+1} \int_{\mathbb{R}^{N}} \rho_{y}\left|\nabla u_{t}\right|^{2} & \leq E_{1}(t)+C_{\mu, \epsilon} \int_{\mathbb{R}^{N}} \rho_{y}\left(|g|^{2}+1\right) \\
& \leq e^{-C_{k, \mu} t} E_{1}(0)+C_{k, \mu, \epsilon} \int_{\mathbb{R}^{N}} \rho_{y}\left(|g|^{2}+1\right),
\end{aligned}
$$

where $E_{1}(\cdot)$ is defined in (4.6). Especially, on the interval $\left[T_{1}, T_{1}+1\right]$ and using (4.13) again, we get that

$$
\begin{aligned}
\int_{T_{1}}^{T_{1}+1} \int_{\mathbb{R}^{N}} \rho_{y}\left|\nabla u_{t}(s)\right|^{2} d x d s & \leq E_{1}\left(T_{1}\right)+C_{k, \mu, \epsilon} \int_{\mathbb{R}^{N}} \rho_{y}\left(|g|^{2}+1\right) \\
& \leq C_{\varrho_{0}}+C_{k, \mu, \epsilon} \int_{\mathbb{R}^{N}} \rho_{y}\left(|g|^{2}+1\right) \quad \text { for all }\left(u_{0}, v_{0}\right) \in B,
\end{aligned}
$$

which, combined with (4.15), implies that for each initial data belonging to $B$, there exists a $\tau_{0} \in\left[T_{1}, T_{1}+1\right]$ (which depends on the initial data) such that

$$
\Lambda\left(\xi_{u}\left(\tau_{0}\right)\right) \leq C_{\varrho_{0}, k, \mu, \epsilon, g} .
$$

Therefore, applying the Gronwall lemma, we deduce from (4.14) that, for all $t \geq T_{1}+1$

$$
\Lambda\left(\xi_{u}(t)\right) \leq \Lambda\left(\xi_{u}\left(\tau_{0}\right)\right) e^{-\left(t-\tau_{0}\right)}+C_{1}+C_{2}\left(e^{-C_{k, \mu}(t-1)}+e^{-(t-1)}\right) E(0)+C_{2} C_{4},
$$

where $C_{4}=C_{k, \mu, \epsilon} \int_{\mathbb{R}^{N}} \rho_{y}\left(|g|^{2}+1\right)$, by which, noticing (4.10), (4.13) and (4.18), we have that

$$
\begin{aligned}
\int_{\mathbb{R}^{N}} & \rho_{y}\left(\left|\nabla u_{t}(t)\right|^{2}+\left|u_{t}(t)\right|^{2}\right) \\
& \leq C_{\varrho_{0}, k, \mu, \epsilon, g}\left(e^{-\left(t-\tau_{0}\right)}+1\right)+C_{2}\left(e^{-C_{k, \mu}(t-1)}+e^{-(t-1)}\right) E(0) .
\end{aligned}
$$

Hence, the conclusion of Lemma 4.2 follows from (4.19) and Theorem 4.1,

Lemma 4.3. Under the assumption of Theorem 3.2, there is a positive constant $\varrho_{2}$ such that for any bounded (in $\mathcal{H}_{0}$ ) subset $B \subset \dot{W}_{l u}^{2,2} \times \dot{L}_{l u}^{2}$, there exists a positive constant $T_{3}=T_{3}(B)$ which depends only on the $\mathcal{H}_{0}$-bound of $B$ such that

$$
\left\|u_{t t}(t)\right\|_{L_{l u}^{2}} \leq \varrho_{2} \quad \text { for all } t \geq T_{3} \text { and }\left(u_{0}, v_{0}\right) \in B \text {. }
$$

Proof. Set $q=u_{t}$ and differentiate (1.1) with respect to time. This yields

$$
q_{t t}+q_{t}-\Delta q_{t}-\Delta q+f^{\prime}(u) u_{t}=0 .
$$

Multiplying (4.20) by $\rho_{y} q_{t}$, we have

$$
\begin{gathered}
\frac{1}{2} \frac{d}{d t}\left(\int_{\mathbb{R}^{N}} \rho_{y}\left|q_{t}\right|^{2}+\int_{\mathbb{R}^{N}} \rho_{y}|\nabla q|^{2}\right)+\int_{\mathbb{R}^{N}} \rho_{y}\left|\nabla q_{t}\right|^{2}+\int_{\mathbb{R}^{N}} \rho_{y}\left|q_{t}\right|^{2} \\
=-\left\langle\nabla q_{t}, \nabla \rho_{y} q_{t}\right\rangle-\left\langle\nabla q, \nabla \rho_{y} q_{t}\right\rangle-\left\langle f^{\prime}(u) u_{t}, \rho_{y} q_{t}\right\rangle,
\end{gathered}
$$

where, by similar calculations, we have

$$
\begin{aligned}
\left|-\left\langle\nabla q_{t}, \nabla \rho_{y} q_{t}\right\rangle\right| & \leq C \sqrt{\eta} \int_{\mathbb{R}^{N}} \rho_{y}\left|\nabla q_{t}\right|^{2}+C \sqrt{\eta} \int_{\mathbb{R}^{N}} \rho_{y}\left|q_{t}\right|^{2}, \\
\left|-\left\langle\nabla q, \nabla \rho_{y} q_{t}\right\rangle\right| & \leq C \sqrt{\eta} \int_{\mathbb{R}^{N}} \rho_{y}|\nabla q|^{2}+C \sqrt{\eta} \int_{\mathbb{R}^{N}} \rho_{y}\left|q_{t}\right|^{2},
\end{aligned}
$$


and applying Proposition 2.4, we have

$$
\left|-\left\langle f^{\prime}(u) u_{t}, \rho_{y} q_{t}\right\rangle\right| \leq \frac{1}{2} \int_{\mathbb{R}^{N}} \rho_{y}\left(\left|q_{t}\right|^{2}+\left|\nabla q_{t}\right|^{2}\right)+\frac{C^{2}}{2}\left\|u_{t}\right\|_{H_{l u}^{1}}^{2}\|u\|_{H_{l u}^{1}}^{\frac{8}{N-2}} .
$$

Taking $\eta$ small enough, for example $C \sqrt{\eta}=\frac{1}{8}$, we get that

$$
\begin{aligned}
& \frac{1}{2} \frac{d}{d t}\left(\int_{\mathbb{R}^{N}} \rho_{y}\left|q_{t}\right|^{2}+\int_{\mathbb{R}^{N}} \rho_{y}|\nabla q|^{2}\right)+\frac{1}{8}\left(\int_{\mathbb{R}^{N}} \rho_{y}\left|q_{t}\right|^{2}+\int_{\mathbb{R}^{N}} \rho_{y}|\nabla q|^{2}\right) \\
& \leq \frac{C^{2}}{2}\left\|u_{t}\right\|_{H_{l u}^{1}}^{2}\|u\|_{H_{l u}^{1}}^{\frac{8}{N-2}}+\frac{1}{8} \int_{\mathbb{R}^{N}} \rho_{y}\left|\nabla u_{t}\right|^{2} .
\end{aligned}
$$

Similarly, applying Theorem 4.1 and Lemma 4.2 , we know that there exists a $T_{2}$ which depends only on the $\mathcal{H}_{0}$-bound of $B$ such that

$$
\|u(t)\|_{H_{l u}^{1}}+\left\|u_{t}(t)\right\|_{H_{l u}^{1}} \leq \varrho_{1} \quad \text { for all } t \geq T_{2} \text { and }\left(u_{0}, v_{0}\right) \in B .
$$

Therefore, from (4.21) we have

$$
\frac{d}{d t}\left(\int_{\mathbb{R}^{N}} \rho_{y}\left|q_{t}\right|^{2}+\int_{\mathbb{R}^{N}} \rho_{y}|\nabla q|^{2}\right)+\frac{1}{4}\left(\int_{\mathbb{R}^{N}} \rho_{y}\left|q_{t}\right|^{2}+\int_{\mathbb{R}^{N}} \rho_{y}|\nabla q|^{2}\right) \leq C_{\varrho_{1}} .
$$

Moreover, integrating (4.12) over $\left[T_{2}, T_{2}+1\right]$, similar to (4.17), we have: for all $\left(u_{0}, v_{0}\right) \in B$,

$$
\int_{T_{2}}^{T_{2}+1} \int_{\mathbb{R}^{N}} \rho_{y}\left|u_{t t}\right|^{2} \leq C_{\varrho_{0}, \varrho_{1},\|g\|_{L_{l u}^{2}}}
$$

which implies that there exists a time $\tau_{1} \in\left[T_{2}, T_{2}+1\right]$ such that

$$
\int_{\mathbb{R}^{N}} \rho_{y}\left|u_{t t}\left(\tau_{1}\right)\right|^{2} d x \leq C_{\varrho_{0}, \varrho_{1},\|g\|_{L_{l u}^{2}}} .
$$

Therefore, using the Gronwall lemma, with (4.22) and (4.24), we know that for any $t \geq T_{2}+1$, the following inequality holds for any $\left(u_{0}, v_{0}\right) \in B$ :

$$
\int_{\mathbb{R}^{N}} \rho_{y}\left(\left|q_{t}(t)\right|^{2}+|\nabla q(t)|^{2}\right) \leq C_{\varrho_{1}, \varrho_{0},\|g\|_{L_{l_{u}}^{2}}}\left(e^{-\frac{\left(t-T_{2}\right)}{4}}+1\right) .
$$

Note that the constant is independent of $y$; then Lemma 4.3 follows immediately.

4.3. Continuity. In the following, we will deduce some continuity with respect to the $H_{\rho}^{1} \times L_{\rho}^{2}$-norm for the solution given in Theorem 3.2 .

Let $\left(u^{i}(t), u_{t}^{i}(t)\right)(i=1,2)$ be the corresponding solution of equation (1.1) to the initial data $\left(u_{0}^{i}, v_{0}^{i}\right) \in \dot{W}_{l u}^{2,2} \times \dot{L}_{l u}^{2}$ and forcing $g_{i}(x) \in \dot{L}_{l u}^{2}$; then their difference $z=u^{1}-u^{2}$ satisfies the equation

$$
z_{t t}+z_{t}-\Delta z_{t}-\Delta z+f\left(u^{1}\right)-f\left(u^{2}\right)=g_{1}-g_{2}
$$


which is equivalent to the equation

$$
\left(z_{t}+\frac{1}{2} z\right)_{t}+\frac{1}{2} z_{t}-\Delta z_{t}-\Delta z+f\left(u^{1}\right)-f\left(u^{2}\right)=g_{1}-g_{2},
$$

with initial data $\left(z(0), z_{t}(0)\right)=\left(u_{0}^{1}-u_{0}^{2}, v_{0}^{1}-v_{0}^{2}\right)$.

Taking $\rho_{y}\left(z_{t}+\frac{1}{2} z\right)$ as the test function, we have

$$
\begin{aligned}
\left\langle\left( z_{t}+\right.\right. & \left.\left.\frac{1}{2} z\right)_{t}, \rho_{y}\left(z_{t}+\frac{1}{2} z\right)\right\rangle+\frac{1}{2}\left\langle z_{t}, \rho_{y}\left(z_{t}+\frac{1}{2} z\right)\right\rangle \\
& \quad-\left\langle\Delta z_{t}, \rho_{y}\left(z_{t}+\frac{1}{2} z\right)\right\rangle-\left\langle\Delta z, \rho_{y}\left(z_{t}+\frac{1}{2} z\right)\right\rangle \\
= & -\left\langle f\left(u^{1}\right)-f\left(u^{2}\right), \rho_{y}\left(z_{t}+\frac{1}{2} z\right)\right\rangle+\left\langle g_{1}-g_{2}, \rho_{y}\left(z_{t}+\frac{1}{2} z\right)\right\rangle
\end{aligned}
$$

recall that $\langle\cdot, \cdot\rangle$ is the usual $L^{2}\left(\mathbb{R}^{N}\right)$-inner product.

In the following, we only estimate the nonlinear term, and the remainder terms can be dealt with in a similar manner as in Theorem 4.1.

Similar to (3.3), we have

$$
\begin{aligned}
\mid-\int_{\mathbb{R}^{N}} \rho_{y} & \left(f\left(u^{1}\right)-f\left(u^{2}\right)\right) z d x \mid \\
\leq & \leq \int_{\mathbb{R}^{N}} \rho_{y}(x)\left(1+\left|u^{1}\right|^{\frac{4}{N-2}}+\left|u^{2}\right|^{\frac{4}{N-2}}\right)|z|^{2} d x \\
& \leq C \int_{\mathbb{R}^{N}} \rho_{y}(s) \int_{B(s, 1)}\left(1+\left|u^{1}\right|^{\frac{4}{N-2}}+\left|u^{2}\right|^{\frac{4}{N-2}}\right)|z|^{2} d x d s \\
& \leq C \int_{\mathbb{R}^{N}} \rho_{y}(x)\left(|\nabla z|^{2}+|z|^{2}\right) d x,
\end{aligned}
$$

where we used Proposition 2.4 and the growth condition (1.3), and the constant $C$ here depends on the constants in Proposition 2.4 and the bounds of initial data $\left\|u_{0}^{i}\right\|_{H_{l u}^{1}}(i=1,2)$. Note that, from the dissipative estimate (4.7), we know that the bounds of $\left\|u^{i}(t)\right\|_{H_{l u}^{1}}(i=1,2)$ for $t \in[0, T]$ depend only on the bounds of initial data.

Similarly, we have

$$
\begin{aligned}
\mid- & \int_{\mathbb{R}^{N}} \rho_{y}\left(f\left(u^{1}\right)-f\left(u^{2}\right)\right) z_{t} d x \mid \\
& \leq C \int_{\mathbb{R}^{N}} \rho_{y}(s) \int_{B(s, 1)}\left(1+\left|u^{1}\right| \frac{4}{N^{-2}}+\left|u^{2}\right| \frac{4}{N^{-2}}\right)|z|\left|z_{t}\right| d x d s \\
& \leq C_{\left\|u_{0}^{i}\right\|_{H_{l u}^{1}}} \int_{\mathbb{R}^{N}} \rho_{y}(s)\left(|z|_{H^{1}(B(s, 1))}\left|z_{t}\right|_{H^{1}(B(s, 1))}\right) d s \\
& \leq C_{\left\|u_{0}^{i}\right\|_{H_{l u}^{1}}, \eta} \int_{\mathbb{R}^{N}} \rho_{y}\left(|\nabla z|^{2}+|z|^{2}\right) d x+\eta \int_{\mathbb{R}^{N}} \rho_{y}\left(\left|\nabla z_{t}\right|^{2}+\left|z_{t}\right|^{2}\right) d x .
\end{aligned}
$$

Therefore, summarizing all of the above estimates into (4.26), we obtain that

$$
\begin{aligned}
& \frac{d}{d t} \int_{\mathbb{R}^{N}} \rho_{y}\left(\left|z_{t}+\frac{1}{2} z\right|^{2}+|\nabla z|^{2}+|z|^{2}\right) \\
& \quad \leq C \int_{\mathbb{R}^{N}} \rho_{y}\left(\left|z_{t}+\frac{1}{2} z\right|^{2}+|\nabla z|^{2}+|z|^{2}\right)+\int_{\mathbb{R}^{N}} \rho_{y}\left|g_{1}-g_{2}\right|^{2},
\end{aligned}
$$


where the constant $C$ depends on the bounds of initial data $\left\|u_{0}^{i}\right\|_{H_{l_{u}}^{1}}(i=1,2)$ and $\eta$, but is independent of $y$. Then using the Gronwall lemma, we get the bounds

$$
\begin{aligned}
\sup _{t \in[0, T]} & \left(\|z(t)\|_{H_{\rho}^{1}}^{2}+\left\|z_{t}(t)\right\|_{L_{\rho}^{2}}^{2}\right) \\
& \leq 4 e^{C T}\left(\|z(0)\|_{H_{\rho}^{1}}^{2}+\left\|z_{t}(0)\right\|_{L_{\rho}^{2}}^{2}\right)+C^{-1}\left\|g_{1}-g_{2}\right\|_{L_{\rho}^{2}}^{2}\left(e^{C T}-1\right), \quad T>0,
\end{aligned}
$$

and

$$
\begin{aligned}
\sup _{t \in[0, T]} & \left(\|z(t)\|_{H_{l u}^{1}}^{2}+\left\|z_{t}(t)\right\|_{L_{l u}^{2}}^{2}\right) \\
& \leq 4 e^{C T}\left(\|z(0)\|_{H_{l u}^{1}}^{2}+\left\|z_{t}(0)\right\|_{L_{l u}^{2}}^{2}\right)+C^{-1}\left\|g_{1}-g_{2}\right\|_{L_{l u}^{2}}^{2}\left(e^{C T}-1\right), \quad T>0 .
\end{aligned}
$$

These justify that, when the initial data varies in a bounded (in $\mathcal{H}_{0}$ ) set $B \subset$ $\dot{W}_{l u}^{2,2} \times \dot{L}_{l u}^{2}$, or the forcing $g$ varies in a bounded (in $L_{l u}^{2}$ ) set $\tilde{B} \subset \dot{L}_{l u}^{2}$, the corresponding solution $\left(u, u_{t}\right)$ is continuous w.r.t. the initial data in the $H_{\rho}^{1} \times L_{\rho}^{2}$-norm and the forcing in the $L_{\rho}^{2}$-norm, uniformly for $t$ varying on bounded subintervals of $[0, \infty)$. The same also holds for the $H_{l u}^{1} \times L_{l u}^{2}$-norm.

\section{Global weak solution}

In this section, based on the a priori estimates and continuities given in section 4 , we will define the weak solution by extending the strong solution (in the sense of Theorem 3.2) to the whole of $H_{l u}^{1} \times L_{l u}^{2}$.

At first, from [11, we give the following definition about the weak $H_{l u}^{1} \times L_{l u}^{2}$ global solution to (1.1)-(1.2), which is slightly different from that in [11]:

Definition 5.1. For each $g \in L_{l u}^{2}$ and any initial data $\left(u_{0}, v_{0}\right) \in H_{l u}^{1} \times L_{l u}^{2}$, the function $\left(u, u_{t}\right) \in L^{\infty}\left([0, \infty), H_{l u}^{1} \times L_{l u}^{2}\right)$ is called a global weak solution to (1.1)(1.2) iff there exist a bounded (in $L_{l u}^{2}$ ) sequence $\left\{g_{n}\right\}_{n=1}^{\infty} \subset \dot{L}_{l u}^{2}$ and a bounded (in $\left.H_{l u}^{1} \times L_{l u}^{2}\right)$ sequence $\left\{\left(u_{n}(0), u_{n t}(0)\right)\right\}_{n=1}^{\infty} \subset \dot{W}_{l u}^{2,2} \times \dot{L}_{l u}^{2}$ satisfying $g_{n} \rightarrow g$ in $L_{\rho}^{2}$ and $\left(u_{n}(0), u_{n t}(0)\right) \rightarrow\left(u_{0}, v_{0}\right)$ in $H_{\rho}^{1} \times L_{\rho}^{2}$, such that the sequence $\left\{\left(u_{n}, u_{n t}\right)\right\}$ of solutions, given in Theorem 3.2 and corresponding to forcing $g_{n}$ and initial data $\left(u_{n}(0), u_{n t}(0)\right)$, converges to $\left(u, u_{t}\right)$ in $\mathcal{C}\left([0, T], H_{\rho}^{1} \times L_{\rho}^{2}\right)$ on the compact interval $[0, T]$ for all $T>0$.

Remark 5.2. The boundedness of $\left\{g_{n}\right\}$ and $\left\{\left(u_{n}(0), u_{n t}(0)\right)\right\}$ in Definition 5.1 are mainly for obtaining the uniform (w.r.t. $n$ ) a priori estimates of approximative solutions.

Proposition $5.3([2,8,26])$. The closure in $H_{\rho}^{r}(r \geq 0)$ of a bounded subset of $H_{U}^{s}$ consists of elements in a bounded subset of $H_{U}^{s}$.

Theorem 5.4. Assume that $f$ satisfies (1.3)-(1.5) and $g \in L_{l u}^{2}$. Then for each $\left(u_{0}, v_{0}\right) \in H_{l u}^{1} \times L_{l u}^{2}$, there is a unique global weak solution to problem (1.1)-(1.2), which defines a continuous (w.r.t. the $H_{\rho}^{1} \times L_{\rho}^{2}$-norm) semigroup $\{S(t)\}_{t \geq 0}$ in $H_{l u}^{1} \times$ $L_{l u}^{2}$.

Proof. The uniqueness follows from (4.29) directly. In the following, we only need to show the existence.

For each $\left(u_{0}, v_{0}\right) \in H_{l u}^{1} \times L_{l u}^{2}$ and each $g \in L_{l u}^{2}$, from Lemma 2.6 we know that there exist $\left(u_{0 n}, v_{0 n}\right) \in \dot{W}_{l u}^{2,2} \times \dot{L}_{l u}^{2}$ with $\left\{\left(u_{0 n}, v_{0 n}\right)\right\}_{n=1}^{\infty}$ bounded in $H_{l u}^{1} \times L_{l u}^{2}$ and $g_{n} \in \dot{L}_{l u}^{2}$ with $\left\{g_{n}\right\}_{n=1}^{\infty}$ bounded in $L_{l u}^{2}$, such that $\left(u_{0 n}, v_{0 n}\right) \rightarrow\left(u_{0}, v_{0}\right)$ w.r.t. the $H_{\rho}^{1} \times L_{\rho}^{2}$-norm and $g_{n} \rightarrow g$ w.r.t. the $L_{\rho}^{2}$-norm. 
From Theorem 3.2 we know that for each $\left(u_{0 n}, v_{0 n}\right) \in \dot{W}_{l u}^{2,2} \times \dot{L}_{l u}^{2}$ and $g_{n} \in$ $\dot{L}_{l u}^{2}$, there is a unique global solution $\left(u_{n}(t), u_{n t}(t)\right)$ given by $\left(u_{n}(t), u_{n t}(t)\right)=$ $\tilde{S}_{g_{n}}(t)\left(u_{0 n}, v_{0 n}\right), n=1,2, \cdots, t \geq 0$, where $\left\{\tilde{S}_{g_{n}}(t)\right\}_{t \geq 0}$ is the $\mathcal{C}^{0}$-semigroup given in Theorem 3.2 corresponding to the forcing $g_{n}$.

Now, for each $g \in L_{l u}^{2}$ and any initial data $\left(u_{0}, v_{0}\right) \in H_{l u}^{1} \times L_{l u}^{2}$, define $S_{g}(t)$ :

$$
S_{g}(t)\left(u_{0}, v_{0}\right)=\lim _{n \rightarrow \infty} \tilde{S}_{g_{n}}(t)\left(u_{n 0}, v_{n 0}\right) \quad \text { in } H_{\rho}^{1} \times L_{\rho}^{2} \quad \text { for every } t \geq 0 .
$$

Then, we claim that:

(i) $S_{g}(t)\left(u_{0}, v_{0}\right) \in H_{l u}^{1} \times L_{l u}^{2}$. This follows from the boundedness (in $H_{l u}^{1} \times L_{l u}^{2}$ ) of $\left\{\left(u_{n 0}, v_{n 0}\right)\right\}_{n=1}^{\infty}$, the boundedness (in $\left.L_{l u}^{2}\right)$ of $\left\{g_{n}\right\}_{n=1}^{\infty}$, Theorem 4.1 and Proposition 5.3 .

(ii) $S_{g}(t)\left(u_{0}, v_{0}\right)$ is independent of the choice of $\left\{\left(u_{n 0}, v_{n 0}\right)\right\}_{n=1}^{\infty}$ and $\left\{g_{n}\right\}_{n=1}^{\infty}$. This follows from (4.29).

(iii) $\left\{S_{g}(t)\right\}_{t \geq 0}$ forms a semigroup on $H_{l u}^{1} \times L_{l u}^{2}$. This follows from the fact that $\left\{\tilde{S}_{g_{n}}(t)\right\}_{t \geq 0}$ is a semigroup for each $g_{n}$ and the continuity estimate (4.29).

(iv) $S_{g}(t): H_{l u}^{1} \times L_{l u}^{2} \rightarrow H_{l u}^{1} \times L_{l u}^{2}$ is continuous w.r.t. the $H_{\rho}^{1} \times L_{\rho}^{2}$-norm. This follows from (4.29).

(v) $\tilde{S}_{g_{n}}(t)\left(u_{n 0}, v_{n 0}\right)$ converges to $S_{g}(t)\left(u_{0}, v_{0}\right)$ in $\mathcal{C}\left([0, T], H_{\rho}^{1}\left(\mathbb{R}^{N}\right) \times L_{\rho}^{2}\left(\mathbb{R}^{N}\right)\right)$ on the compact interval $[0, T]$ for all $T>0$. This follows from (4.29).

(vi) $S_{g}(t)=\tilde{S}_{g}(t)$ on $\dot{H}_{l u}^{2} \times \dot{L}_{l u}^{2}$ for every $t \geq 0$ provided that $g \in \dot{L}_{l u}^{2}$.

Hence, $S_{g}(t)\left(u_{0}, v_{0}\right)$ is the unique global weak solution of (1.1)-(1.2) corresponding to the initial data $\left(u_{0}, v_{0}\right)$, where $S_{g}(t)\left(u_{0}, v_{0}\right) \in L^{\infty}\left([0, \infty), H_{l u}^{1} \times L_{l u}^{2}\right)$ follows from Theorem 4.1 and Proposition 5.3 .

Remark 5.5. In Theorem 5.4, we use the a priori estimate (4.29) to guarantee the existence of a weak global $H_{l u}^{1} \times L_{l u}^{2}$ solution semigroup $\{S(t)\}_{t \geq 0}$, which indeed is an extension of $\{\tilde{S}(t)\}_{t \geq 0}$ w.r.t. the weighted norm to phase space $H_{l u}^{1} \times L_{l u}^{2}$ with the forcing $g \in L_{l u}^{2}$.

On the other hand, as shown in (4.30), we also have the "strong" continuity w.r.t. the norm of uniform space. Hence, if we extend $\{\tilde{S}(t)\}_{t \geq 0}$ with the uniform space norm, that is, we pass the limit in (5.1) with the uniform space norm, then we can also uniquely define a continuous semigroup, whose phase space is $\dot{H}_{l u}^{1} \times \dot{L}_{l u}^{2}$ and with the forcing $g \in \dot{L}_{l u}^{2}$, which is just the semigroup defined in Cholewa \& Dlotko 12 .

Remark 5.6. In particular, (4.30) also implies the Lipschitz continuity of $\{S(t)\}_{t>0}$. That is, given any $R>0$ and any two initial data $z_{1}, z_{2} \in \mathcal{H}_{0}$ with $\left\|z_{i}\right\|_{\mathcal{H}_{0}} \leq R$, $i=1,2$, there holds

$$
\left\|S(t) z_{1}-S(t) z_{2}\right\|_{\mathcal{H}_{0}} \leq e^{C_{R} t}\left\|z_{1}-z_{2}\right\|_{\mathcal{H}_{0}}, \quad \forall t \geq 0
$$

Remark 5.7. In this paper, we define the weak solution semigroup by extending the strong continuous analytic semigroup generated by a sectorial operator, which is different from some previous literatures, e.g., the solution in the sense of distribution given in 16, 34. Although they start in a different manner, these two kinds of solutions are indeed the same.

\section{Hereafter, we always take the following assumption.}

Assumption I. $f$ satisfies (1.3)-(1.5), $g \in L_{l u}^{2}$ and $\{S(t)\}_{t \geq 0}$ is the semigroup corresponding to the global weak solutions of (1.1) $-(1.2)$ in $H_{l u}^{1} \times L_{l u}^{2}$. 


\section{Decomposition of the Equations}

For the nonlinear term $f$, following the idea in [1, we have the following properties.

For a $\mathcal{C}^{1}$-function satisfying (1.3)-(1.5), the following decomposing properties hold:

there are constants $C>0$ and $\gamma$ satisfying $0<\gamma<q+1$ such that $f$ can be decomposed as $f=f_{0}+f_{1}$ with $f_{0}, f_{1} \in \mathcal{C}^{1}(\mathbb{R})$ satisfying

$$
\left\{\begin{array}{ccc}
1 . & \left|f_{0}(s)\right| \leq C\left(|s|+|s|^{q+1}\right) \quad \forall s \in \mathbb{R}, \\
2 . & f_{0}(s) s \geq 0 \quad \forall s \in \mathbb{R}, \\
3 . & \exists \tilde{k}_{0} \geq 1, \tilde{\mu}_{0}>0 \text { such that } \forall \tilde{\mu} \in\left(0, \tilde{\mu}_{0}\right], \quad \exists C_{\tilde{\mu}} \in \mathbb{R}, \\
& \tilde{k}_{0} F_{0}(s)+\tilde{\mu} s^{2}-C_{\tilde{\mu}} \leq s f_{0}(s) \quad \forall s \in \mathbb{R},
\end{array}\right.
$$

and

$$
\left\{\begin{array}{c}
\text { 1. } \quad\left|f_{1}(s)\right| \leq C\left(1+|s|^{\gamma}\right) \quad \forall s \in \mathbb{R} \text { with some } \gamma<q+1, \\
\text { 2. } \quad \exists \tilde{k}_{1} \geq 1, \tilde{\mu}_{1}>0 \text { such that } \forall \tilde{\mu} \in\left(0, \tilde{\mu}_{1}\right], \exists C_{\tilde{\mu}} \in \mathbb{R}, \\
\tilde{k}_{1} F_{1}(s)+\tilde{\mu} s^{2}-C_{\tilde{\mu}} \leq s f_{1}(s) \quad \forall s \in \mathbb{R}, \\
\text { 3. } \exists \tilde{c}^{\prime} \in \mathbb{R},-\tilde{c}^{\prime} \leq F_{1}(s) \quad \forall s \in \mathbb{R},
\end{array}\right.
$$

where $F_{i}(s)=\int_{0}^{s} f_{i}(\tau) d \tau, i=0,1$.

For example, from (1.4)-(1.5) we know that there is an $M>0$ such that

$$
k F(s)+\frac{\mu_{1}}{2} s^{2}-C_{\mu_{1}} \geq 0 \text { for all }|s| \geq M .
$$

Taking a cutoff function $\psi(s)$ satisfies

$$
\psi(s)= \begin{cases}1, & |s| \geq 2 M \\ 0, & |s| \leq M\end{cases}
$$

Set

$$
f_{0}(s)=\psi(s)\left(f(s)-\frac{\mu_{1}}{2} s\right) \text { and } f_{1}(s)=f(s)-f_{0}(s)
$$

for all $s \in \mathbb{R}$.

Then, we can verify that the above $f_{0}, f_{1}$ satisfies (6.1)-(6.2) respectively for some proper constants.

In order to obtain the latter regularity estimates, as in [27, 35, we decompose the solution $S(t)\left(u_{0}, v_{0}\right)=\left(u(t), u_{t}(t)\right)$ into the sum

$$
S(t)\left(u_{0}, v_{0}\right)=D(t)\left(u_{0}, v_{0}\right)+K(t)\left(u_{0}, v_{0}\right),
$$

where $D(t)\left(u_{0}, v_{0}\right)=\left(v(t), v_{t}(t)\right)$ and $K(t)\left(u_{0}, v_{0}\right)=\left(w(t), w_{t}(t)\right)$ solve the following equations respectively:

$$
\left\{\begin{array}{l}
v_{t t}+v_{t}-\Delta v_{t}-\Delta v+f_{0}(v)+v=0 \\
\left(v(0), v_{t}(0)\right)=\left(u_{0}, v_{0}\right)
\end{array}\right.
$$

and

$$
\left\{\begin{array}{l}
w_{t t}+w_{t}-\Delta w_{t}-\Delta w+f(u)-f_{0}(v)-v=g(x) \\
\left(w(0), w_{t}(0)\right)=0
\end{array}\right.
$$


Note that $\{D(t)\}_{t \geq 0}$ also forms a semigroup, but $\{K(t)\}_{t \geq 0}$ may not, and we denote

$$
\sigma=\min \left\{\frac{1}{4}, \frac{N+2-(N-2) \gamma}{2}\right\}
$$

where $\gamma$ is given in (6.2).

\section{A PRIORI ESTIMATES}

In this section, we will establish some a priori estimates about the solutions of equations (6.4) and (6.5), which are the basis of our latter analysis.

Note that, according to the definition of weak solutions given in Definition 5.1 (especially the concrete expression (5.1), the continuity estimate (4.29) and Proposition [5.3), we know that for the a priori estimates of weak solutions, it suffices to deal first with the (approximative) strong solutions of (1.1)-(1.2), and then by passing the limit (as (5.1)) we can deduce the same estimates for weak solutions.

Hence, hereafter, all a priori estimates in this paper are in the sense of strong solutions.

First, for convenience, we always denote by $B_{0}$ a bounded $\left(\mathcal{H}_{0}, \mathcal{H}_{0}\right)$-absorbing set (obtained in Theorem 4.1) of $\{S(t)\}_{t \geq 0}$ and set

$$
B_{1}=\bigcup_{t \geq T_{B_{0}}} S(t) B_{0}
$$

where $T_{B_{0}}$ is the maximum time obtained in Theorem 4.1 and Lemmas 4.2 and 4.3 corresponding to $B_{0}$. Then, $B_{1}$ is also a bounded $\left(\mathcal{H}_{0}, H_{l u}^{1} \times H_{l u}^{1}\right)$-absorbing set, and is positive invariant. Moreover, from the proof of Lemma 4.3 and the uniqueness of solutions, we also know that: for any initial data $\left(u_{0}, v_{0}\right) \in B_{1}$, the following estimate holds:

$$
\|u(t)\|_{H_{l u}^{1}}^{2}+\left\|u_{t}(t)\right\|_{H_{l u}^{1}}^{2}+\left\|u_{t t}(t)\right\|_{L_{l u}^{2}}^{2} \leq C_{\varrho_{0}, \varrho_{1}, \varrho_{2}} \quad \text { for all } t \geq 0 .
$$

Second, from the conditions (6.1) of $f_{0}$, we can repeat the proofs of Theorem 4.1 and Lemmas 4.2 and 4.3 to get the following dissipation estimates about the semigroup $\{D(t)\}_{t \geq 0}$ :

Lemma 7.1. Under Assumption $I$ and $f_{0}$ satisfying (6.1), the semigroup $\{D(t)\}_{t \geq 0}$ generated by the solutions of equation (6.4) satisfies the following estimates:

(i) there exists a positive constant $\varrho_{v}$ such that for any bounded (in $\mathcal{H}_{0}$ ) set $B$, there is a $T=T\left(\|B\|_{\mathcal{H}_{0}}\right)$ such that

$$
\|v(t)\|_{H_{l u}^{1}}^{2}+\left\|v_{t}(t)\right\|_{H_{l u}^{1}}^{2}+\left\|v_{t t}(t)\right\|_{L_{l u}^{2}}^{2} \leq \varrho_{v}
$$

for all $t \geq T$ and any $\left(u_{0}, v_{0}\right) \in B$;

(ii) $D(t)$ maps the bounded set of $\mathcal{H}_{0}$ to be a uniformly (w.r.t. time $t$ ) bounded set; that is, for any $\left(u_{0}, v_{0}\right) \in \mathcal{H}_{0}$,

$$
\left\|D(t)\left(u_{0}, v_{0}\right)\right\|_{\mathcal{H}_{0}}^{2} \leq Q\left(\left\|\left(u_{0}, v_{0}\right)\right\|_{\mathcal{H}_{0}}\right) \quad \text { for all } t \geq 0 .
$$

Proof. (i) is a repeat of Theorem 4.1 and Lemmas 4.2 and 4.3 . (ii) is a direct result of (4.7). 
Third, from the proof of Lemma 4.2. we can also see that if the initial data is more regular, e.g., $\left(u_{0}, v_{0}\right) \in B_{1}$, then one can get a further result, for example:

Lemma 7.2. Under Assumption I and $f_{0}$ satisfying (6.1), there exists $\varrho_{3}$ which depends on the $H_{l u}^{1} \times H_{l u}^{1}$-bound of $B_{1}$ such that

$$
\left\|v_{t}(t)\right\|_{H_{l u}^{1}}^{2} \leq \varrho_{3} \text { for all } t \geq 0 \text { and all }\left(u_{0}, v_{0}\right) \in B_{1},
$$

where $B_{1}$ is given in (7.1) and $\left(v(t), v_{t}(t)\right)=D(t)\left(u_{0}, v_{0}\right)$ is the solution of (6.4).

Proof. This is a repeat of the proof of Lemma 4.2 word by word: we only need to apply the Gronwall lemma to a similar inequality as (4.14) for $v(t)$, and note that now we can state that $\tau_{0}$ (in (4.18) ) equals 0 since the initial data $\left(u_{0}, v_{0}\right)$ now belongs to $H_{l u}^{1} \times H_{l u}^{1}$.

As a direct result of (7.5) and Lemmas 4.2 and 7.1, for the solutions $\left(w(t), w_{t}(t)\right)$ $=K(t)\left(u_{0}, v_{0}\right)$ of equation (6.5), we have that there is a $\varrho_{4}$ which depends on the $H_{l u}^{1} \times H_{l u}^{1}$-bound of $B_{1}$ such that

$$
\|w(t)\|_{H_{l u}^{1}}^{2}+\left\|w_{t}(t)\right\|_{H_{l u}^{1}}^{2} \leq \varrho_{4} \quad \text { for all } t \geq 0 \text { and all }\left(u_{0}, v_{0}\right) \in B_{1} .
$$

Furthermore, as in 5, 20, 27, 29, 30, for the solution of (6.4) we have the following exponential decay result:

Lemma 7.3. Under Assumption I and $f_{0}$ satisfying (6.1), the solutions of equation (6.4) satisfy the following estimate: there exists a constant $k_{0}$ such that for every $t \geq 0$,

$$
\left\|D(t)\left(u_{0}, v_{0}\right)\right\|_{\mathcal{H}_{0}}^{2}=\|v(t)\|_{H_{l u}^{1}}^{2}+\left\|v_{t}(t)\right\|_{L_{l u}^{2}}^{2} \leq Q_{1}\left(\left\|\left(u_{0}, v_{0}\right)\right\|_{\mathcal{H}_{0}}\right) e^{-k_{0} t}
$$

where $Q_{1}(\cdot)$ is an increasing function on $[0, \infty)$ and $k_{0}$ depends on the $\mathcal{H}_{0}$-bound of $\left(u_{0}, v_{0}\right)$.

Proof. Multiplying (6.4) by $\rho_{y}\left(v_{t}+\frac{1}{2} v\right)$, we get that (note again that here and after we will only give formal calculations for strong solutions which are obtained in Theorem 3.2, and for the weak solutions that are obtained in Theorem 5.4 we deduce by a limit approximation)

$$
\begin{array}{r}
\left\langle\left(v_{t}+\frac{1}{2} v\right)_{t}, \rho_{y}\left(v_{t}+\frac{1}{2} v\right)\right\rangle+\frac{1}{2}\left\langle v_{t}+\frac{1}{2} v, \rho_{y}\left(v_{t}+\frac{1}{2} v\right)\right\rangle-\left\langle\Delta v_{t}, \rho_{y}\left(v_{t}+\frac{1}{2} v\right)\right\rangle \\
-\left\langle\Delta v, \rho_{y}\left(v_{t}+\frac{1}{2} v\right)\right\rangle+\left\langle f_{0}(v), \rho_{y}\left(v_{t}+\frac{1}{2} v\right)\right\rangle+\frac{3}{4}\left\langle v, \rho_{y}\left(v_{t}+\frac{1}{2} v\right)\right\rangle=0 .
\end{array}
$$

By some standard calculations, we have

$$
\begin{aligned}
\frac{1}{2} \frac{d}{d t} & \left(\int_{\mathbb{R}^{N}} \rho_{y}\left|v_{t}+\frac{1}{2} v\right|^{2}+\frac{3}{2} \int_{\mathbb{R}^{N}} \rho_{y}|\nabla v|^{2}+\frac{3}{4} \int_{\mathbb{R}^{N}} \rho_{y}|v|^{2}+2 \int_{\mathbb{R}^{N}} \rho_{y} F_{0}(v)\right) \\
& +\frac{1}{2} \int_{\mathbb{R}^{N}} \rho_{y}\left|v_{t}+\frac{1}{2} v\right|^{2}+\int_{\mathbb{R}^{N}} \rho_{y}\left|\nabla v_{t}\right|^{2}+\frac{1}{2} \int_{\mathbb{R}^{N}} \rho_{y}|\nabla v|^{2}+\frac{1}{2} \int_{\mathbb{R}^{N}} \rho_{y} f_{0}(v) v \\
& +\frac{3}{8} \int_{\mathbb{R}^{N}} \rho_{y}|v|^{2}+\int_{\mathbb{R}^{N}} \nabla v_{t} \nabla \rho_{y}\left(v_{t}+\frac{1}{2} v\right)+\int_{\mathbb{R}^{N}} \nabla v \nabla \rho_{y}\left(v_{t}+\frac{1}{2} v\right)=0,
\end{aligned}
$$

where

$$
\left|\int_{\mathbb{R}^{N}} \nabla v_{t} \nabla \rho_{y}\left(v_{t}+\frac{1}{2} v\right)\right| \leq C \sqrt{\eta} \int_{\mathbb{R}^{N}} \rho_{y}\left(\left|\nabla v_{t}\right|^{2}+\left|v_{t}+\frac{1}{2} v\right|^{2}\right)
$$


and

$$
\left|\int_{\mathbb{R}^{N}} \nabla v \nabla \rho_{y}\left(v_{t}+\frac{1}{2} v\right)\right| \leq C \sqrt{\eta} \int_{\mathbb{R}^{N}} \rho_{y}\left(|\nabla v|^{2}+\left|v_{t}+\frac{1}{2} v\right|^{2}\right) .
$$

Moreover, from (6.1 $)_{1}$ and $(6.1)_{2}$, we have that

$$
\int_{\mathbb{R}^{N}} \rho_{y} F_{0}(v) \leq C \int_{\mathbb{R}^{N}} \rho_{y}\left(|v|^{2}+|v|^{\frac{2 N}{N-2}}\right)
$$

and

$$
F_{0}(v) \geq 0 \quad \forall v \in \mathbb{R} ; \quad f_{0}(v) v \geq 0 \quad \forall v \in \mathbb{R} .
$$

Now, applying Proposition 2.4 (several times), we have

$$
\int_{\mathbb{R}^{N}} \rho_{y}(x)|v(x)|^{\frac{2 N}{N-2}} d x \leq C\|v\|_{H_{l u}^{1}}^{\frac{4}{N-2}} \int_{\mathbb{R}^{N}} \rho_{y}(x)\left(|v(x)|^{2}+|\nabla v(x)|^{2}\right) d x,
$$

which, combined with (7.8) and (7.4), implies that

$$
\int_{\mathbb{R}^{N}} \rho_{y} F_{0}(v(t)) \leq C_{\left\|\left(u_{0}, v_{0}\right)\right\|_{\mathcal{H}_{0}}} \int_{\mathbb{R}^{N}} \rho_{y}\left(|v(t)|^{2}+|\nabla v(t)|^{2}\right) \quad \text { for all } t \geq 0 .
$$

Hence, choosing $\eta$ small enough, we can obtain that there exists a $k_{0}>0$ (which is small and depends on the $\mathcal{H}_{0}$-bound of initial data (e.g., by (7.9) and (7.10) ) such that

$$
\begin{aligned}
& \frac{d}{d t}\left(\int_{\mathbb{R}^{N}} \rho_{y}\left|v_{t}+\frac{1}{2} v\right|^{2}+\frac{3}{2} \int_{\mathbb{R}^{N}} \rho_{y}|\nabla v|^{2}+\frac{3}{4} \int_{\mathbb{R}^{N}} \rho_{y}|v|^{2}+2 \int_{\mathbb{R}^{N}} \rho_{y} F_{0}(v)\right)+\frac{1}{2} \int_{\mathbb{R}^{N}} \rho_{y}\left|\nabla v_{t}\right|^{2} \\
& \quad+k_{0}\left(\int_{\mathbb{R}^{N}} \rho_{y}\left|v_{t}+\frac{1}{2} v\right|^{2}+\frac{3}{2} \int_{\mathbb{R}^{N}} \rho_{y}|\nabla v|^{2}+\frac{3}{4} \int_{\mathbb{R}^{N}} \rho_{y}|v|^{2}+2 \int_{\mathbb{R}^{N}} \rho_{y} F_{0}(v)\right) \leq 0 .
\end{aligned}
$$

Then, applying the Gronwall lemma and noticing $F_{0}(v) \geq 0$ again, we obtain that

$$
\int_{\mathbb{R}^{N}} \rho_{y}\left|v_{t}(t)+\frac{1}{2} v(t)\right|^{2}+\frac{3}{2} \int_{\mathbb{R}^{N}} \rho_{y}|\nabla v(t)|^{2}+\frac{3}{4} \int_{\mathbb{R}^{N}} \rho_{y}|v(t)|^{2} \leq Q\left(\left\|\xi_{v}(0)\right\|_{\mathcal{H}_{0}}^{2}\right) e^{-k_{0} t}
$$

For the solution of equation (6.5), we have the following regularity result:

Lemma 7.4. Under Assumption I and the decomposition (6.1)-(6.2) of $f$, the solutions of equation (6.5) satisfy the following estimate: for any initial data $\left(u_{0}, v_{0}\right) \in$ $B_{1}$, there exists a constant $k_{1}$ such that for every $t \geq 0$,

$$
\left\|w_{t}(t)\right\|_{H_{U}^{\sigma}}^{2}+\|w(t)\|_{H_{U}^{1+\sigma}}^{2} \leq Q_{2}\left(\left\|\left(u_{0}, v_{0}\right)\right\|_{H_{l u}^{1} \times H_{l u}^{1}},\|g\|_{L_{l u}^{2}}\right) e^{k_{1} t}
$$

where $\sigma=\min \left\{\frac{1}{4}, \frac{N+2-(N-2) \gamma}{2}\right\}$ is given in (6.6), $Q_{2}(\cdot)$ is an increasing function on $[0, \infty)$ and $k_{1}$ depends on $\left\|\left(u_{0}, v_{0}\right)\right\|_{H_{l u}^{1} \times H_{l u}^{1}},\|g\|_{L_{l u}^{2}}$.

The aim of this lemma is to obtain a higher regularity of $\left(w(t), w_{t}(t)\right)$; naturally, we will use the intermediate spaces technique as used in previous references; e.g. [1, 6, 13, 27. 
Proof of Lemma 7.4. Let $\theta$ be a smooth function satisfying $0 \leq \theta(s) \leq 1$ for $s \in$ $[0, \infty)$, and

$$
\theta(s)=1 \text { for } 0 \leq s \leq \frac{1}{2} ; \quad \theta(s)=0 \text { for } s \geq 1 .
$$

Set $\theta_{y}(x)=\theta(|x-y|)$. Multiplying (6.5) by $\theta_{y} A^{\sigma}\left(\theta_{y} w_{t}\right)$, we have

$$
\begin{aligned}
\frac{1}{2} \frac{d}{d t} & \int_{\mathbb{R}^{N}}\left|A^{\frac{\sigma}{2}}\left(\theta_{y} w_{t}\right)\right|^{2}+\int_{\mathbb{R}^{N}}\left|A^{\frac{\sigma}{2}}\left(\theta_{y} w_{t}\right)\right|^{2}-\left\langle\Delta w_{t}, \theta_{y} A^{\sigma}\left(\theta_{y} w_{t}\right)\right\rangle \\
& -\left\langle\Delta w, \theta_{y} A^{\sigma}\left(\theta_{y} w_{t}\right)\right\rangle+\left\langle f(u)-f(v)+f_{1}(v), \theta_{y} A^{\sigma}\left(\theta_{y} w_{t}\right)\right\rangle \\
& =\left\langle g+v, \theta_{y} A^{\sigma}\left(\theta_{y} w_{t}\right)\right\rangle .
\end{aligned}
$$

We deal with each term above one by one:

$$
-\left\langle\Delta w_{t}, \theta_{y} A^{\sigma}\left(\theta_{y} w_{t}\right)\right\rangle=\int_{\mathbb{R}^{N}}\left|A^{\frac{\sigma+1}{2}}\left(\theta_{y} w_{t}\right)\right|^{2}+\left\langle\Delta \theta_{y} w_{t}+2 \nabla \theta_{y} \cdot \nabla w_{t}, A^{\sigma}\left(\theta_{y} w_{t}\right)\right\rangle,
$$

where, since $\sigma<\frac{1}{2}$, by the continuous embedding, we have

$$
\begin{aligned}
\left|\left\langle\Delta \theta_{y} w_{t}+2 \nabla \theta_{y} \cdot \nabla w_{t}, A^{\sigma}\left(\theta_{y} w_{t}\right)\right\rangle\right| & \leq C\left\|w_{t}(t)\right\|_{H_{U}^{1}}\left(\int_{\mathbb{R}^{N}}\left|A^{\sigma}\left(\theta_{y} w_{t}\right)\right|^{2}\right)^{\frac{1}{2}} \\
& \leq C\left\|w_{t}(t)\right\|_{H_{U}^{1}}^{2} .
\end{aligned}
$$

Similarly,

$$
-\left\langle\Delta w, \theta_{y} A^{\sigma}\left(\theta_{y} w_{t}\right)\right\rangle=\frac{1}{2} \frac{d}{d t} \int_{\mathbb{R}^{N}}\left|A^{\frac{\sigma+1}{2}}\left(\theta_{y} w\right)\right|^{2}+\left\langle\Delta \theta_{y} w+2 \nabla \theta_{y} \cdot \nabla w, A^{\sigma}\left(\theta_{y} w_{t}\right)\right\rangle
$$

and

$$
\begin{aligned}
\left|\left\langle\Delta \theta_{y} w+2 \nabla \theta_{y} \cdot \nabla w, A^{\sigma}\left(\theta_{y} w_{t}\right)\right\rangle\right| & \leq C\|w(t)\|_{H_{U}^{1}}\left(\int_{\mathbb{R}^{N}}\left|A^{\sigma}\left(\theta_{y} w_{t}\right)\right|^{2}\right)^{\frac{1}{2}} \\
& \leq C\|w(t)\|_{H_{U}^{1}}\left\|w_{t}(t)\right\|_{H_{U}^{1}} .
\end{aligned}
$$

On the other hand, since $\sigma \leq \frac{N+2-(N-2) \gamma}{2}$, by $(6.2) 1$ we have

$$
\begin{aligned}
& \left|\left\langle f_{1}(v), \theta_{y} A^{\sigma}\left(\theta_{y} w_{t}\right)\right\rangle\right| \\
& \leq C \int_{\mathbb{R}^{N}} \theta_{y}\left(1+|v|^{\gamma}\right)\left|A^{\sigma}\left(\theta_{y} w_{t}\right)\right| \\
& \leq C\left(\int_{\mathbb{R}^{N}}\left|A^{\sigma}\left(\theta_{y} w_{t}\right)\right|^{\frac{2 N}{N-2+2 \sigma}}\right)^{\frac{N-2+2 \sigma}{2 N}}\left(\int_{B(y, 1)}\left|\left(1+|v|^{\gamma}\right)\right|^{\frac{2 N}{N+2-2 \sigma}}\right)^{\frac{N+2-2 \sigma}{2 N}} \\
& \leq C\left(1+\|v\|_{H_{U}^{1}}^{\gamma}\right)\left(\left\|\theta_{y} w_{t}\right\|_{L^{2}}+\left\|A^{\frac{\sigma+1}{2}}\left(\theta_{y} w_{t}\right)\right\|_{L^{2}}\right) .
\end{aligned}
$$

By virtue of (1.3),

$$
\begin{aligned}
& \left|\left\langle f(u)-f(v), \theta_{y} A^{\sigma}\left(\theta_{y} w_{t}\right)\right\rangle\right| \\
& \leq C \int_{\mathbb{R}^{N}} \theta_{y}|w|\left(1+|u|^{\frac{4}{N-2}}+|v|^{\frac{4}{N-2}}\right)\left|A^{\sigma}\left(\theta_{y} w_{t}\right)\right| \\
& \leq C\left(\int_{\mathbb{R}^{N}}\left|A^{\sigma}\left(\theta_{y} w_{t}\right)\right|^{p_{1}}\right) \frac{1}{p_{1}}\left(\int_{\mathbb{R}^{N}}\left|\theta_{y} w\right|^{p_{2}}\right) \frac{1}{p_{2}}\left(\int_{B(y, 1)}\left(1+|u|^{\frac{4}{N-2}}+|v|^{\frac{4}{N-2}}\right)^{\frac{2 N}{4}}\right) \frac{4}{2 N} \\
& \leq C\left(\left\|\theta_{y} w_{t}\right\|_{L^{2}}+\left\|A^{\frac{\sigma+1}{2}}\left(\theta_{y} w_{t}\right)\right\|_{L^{2}}\right)\left(\left\|\theta_{y} w\right\|_{L^{2}}+\left\|A^{\frac{\sigma+1}{2}}\left(\theta_{y} w\right)\right\|_{L^{2}}\right), \\
& \text { where } p_{1}=\frac{2 N}{N-2+2 \sigma}, p_{2}=\frac{2 N}{N-2-2 \sigma} \text { and } \sigma \leq \frac{1}{4}<\frac{N-2}{2} .
\end{aligned}
$$


Finally, noting $\sigma<\frac{1}{2}$ again, we have

$$
\left|\left\langle g+v, \theta_{y} A^{\sigma}\left(\theta_{y} w_{t}\right)\right\rangle\right| \leq\|g+v\|_{L_{U}^{2}}\left\|w_{t}(t)\right\|_{H_{U}^{1}} .
$$

Therefore,

$$
\begin{aligned}
& \frac{1}{2} \frac{d}{d t}\left(\int_{\mathbb{R}^{N}}\left|A^{\frac{\sigma}{2}}\left(\theta_{y} w_{t}\right)\right|^{2}+\int_{\mathbb{R}^{N}}\left|A^{\frac{\sigma+1}{2}}\left(\theta_{y} w\right)\right|^{2}\right) \\
& \quad \leq k_{1}\left(\int_{\mathbb{R}^{N}}\left|A^{\frac{\sigma}{2}}\left(\theta_{y} w_{t}\right)\right|^{2}+\int_{\mathbb{R}^{N}}\left|A^{\frac{\sigma+1}{2}}\left(\theta_{y} w\right)\right|^{2}\right)+C_{g,\|v\|_{H_{U}^{1}},\left\|w_{t}(t)\right\|_{H_{U}^{1}},\|w(t)\|_{H_{U}^{1}}},
\end{aligned}
$$

where $k_{1}$ depends on $C,\|v\|_{H_{U}^{1}},\left\|w_{t}(t)\right\|_{H_{U}^{1}},\|w(t)\|_{H_{U}^{1}}$. Note that, from Lemma 7.1 and (7.6), we know that $\left\|w_{t}(t)\right\|_{H_{l u}^{1}}$ and $\|w(t)\|_{H_{l u}^{1}}$ only depend on $\left\|\left(u_{0}, v_{0}\right)\right\|_{H_{l u}^{1} \times H_{l u}^{1}}$ and $\|g\|_{L_{l u}^{2}}$.

Hence, applying the Gronwall lemma, we get that

$$
\int_{\mathbb{R}^{N}}\left|A^{\frac{\sigma}{2}}\left(\theta_{y} w_{t}\right)\right|^{2}+\int_{\mathbb{R}^{N}}\left|A^{\frac{\sigma+1}{2}}\left(\theta_{y} w\right)\right|^{2} \leq Q_{2}\left(\left\|\left(u_{0}, v_{0}\right)\right\|_{H_{l u}^{1} \times H_{l u}^{1}},\|g\|_{L_{l u}^{2}}\right)\left(e^{k_{1} t}-1\right) .
$$

After taking the supremum with respect to $y \in \mathbb{R}^{N}$, we indeed get that

$$
\left\|A^{\frac{1+\sigma}{2}} w(t)\right\|_{L_{U}^{2}}^{2}+\left\|A^{\frac{\sigma}{2}} w_{t}(t)\right\|_{L_{U}^{2}}^{2} \leq Q_{2}\left(\left\|\left(u_{0}, v_{0}\right)\right\|_{H_{l u}^{1} \times H_{l u}^{1}},\|g\|_{L_{l u}^{2}}\right) e^{k_{1} t},
$$

which, combined with Lemma 2.2 and (7.6), implies (7.13).

\section{AsYmptotic REgUlarity of THE SOLUtions}

In this section, we will show by some bootstrap arguments that there indeed exists a more regular (bounded in $H_{U}^{2} \times H_{U}^{1}$ ) set $\mathcal{B}_{1}$ which attracts exponentially (w.r.t. the $\mathcal{H}_{0}$-norm) any $\mathcal{H}_{0}$-bounded set.

Similar to that in Zelik [35], based on Lemma 7.3] and Lemma 7.4, for the solution $\left(u(t), u_{t}(t)\right)$ started from the initial data which belongs to $B_{1}$ (given in (7.1)), we can decompose it as follows:

Lemma 8.1. Under Assumption I, let $\left(u(t), u_{t}(t)\right)$ be the solution of (1.1)-(1.2) corresponding to the initial data $z_{0}=\left(u_{0}, v_{0}\right) \in B_{1}$. Then, for every $\varepsilon>0$ there exist positive constants $C_{\varepsilon}$ (which depends only on the $\mathcal{H}_{0}$-bound of $B_{1}$ ) and $K_{\varepsilon}$ (which depends on $\varepsilon$ and the $H_{U}^{1} \times H_{U}^{1}$-bound of $B_{1}$ ), such that

$$
u(t)=v_{1}(t)+w_{1}(t) \quad \text { for all } t \geq 0,
$$

where $v_{1}(t), w_{1}(t)$ satisfy the following estimates:

$$
\left\|w_{1}(t)\right\|_{H_{U}^{1+\sigma}}^{2} \leq K_{\varepsilon} \quad \text { for all } t \geq 0
$$

and, for every $t \geq s \geq 0$,

$$
\int_{s}^{t}\left\|v_{1}(\nu)\right\|_{H_{U}^{1}}^{2} d \nu \leq \varepsilon(t-s)+C_{\varepsilon} .
$$

The proof of this lemma is similar to that in 35 .

Proof. From the definition of $B_{1}$ (in (7.1)), we know that

$$
\sup _{t \geq 0}\left\|S(t) z_{0}\right\|_{\mathcal{H}_{0}}^{2} \leq \varrho_{1} \quad \text { for all } z_{0} \in B_{1},
$$

where $\varrho_{1}$ is given in Theorem 4.1 . 
Now, taking $T \geq \frac{1}{k_{0}} \ln \frac{Q_{1}\left(\varrho_{1}\right)}{\varepsilon}$ (where $Q_{1}(\cdot)$ is the function in Lemma 7.3), and in every interval $[m T,(m+1) T), m=1,2, \cdots$, we set

$$
v_{1}(t)=v(t) \quad \text { and } \quad w_{1}(t)=w(t)
$$

where $v(t)$ and $w(t)$ are the solutions of (6.4) and (6.5) respectively in the interval $[(m-1) T,(m+1) T)$ with the initial data $\left(v((m-1) T), v_{t}((m-1) T)\right)=$ $\left(u((m-1) T), u_{t}((m-1) T)\right)$ and $\left(w((m-1) T), w_{t}((m-1) T)\right)=(0,0)$.

And in interval $[0, T)$, we set

$$
v_{1}(t)=v(t) \quad \text { and } \quad w_{1}(t)=w(t)
$$

where $v(t)$ and $w(t)$ are the solutions of (6.4) and (6.5) respectively in the interval $[0, T)$ with the initial data $\left(v(0), v_{t}(0)\right)=z_{0}$ and $\left(w(0), w_{t}(0)\right)=(0,0)$.

Then from Lemma 7.3 we have

$$
\int_{s}^{t}\left\|v_{1}(\nu)\right\|_{H_{U}^{1}}^{2} d \nu \leq \varepsilon(t-s)+\chi_{[0, T)}(s) Q_{1}\left(\varrho_{1}\right) \text { for all } t \geq s \geq 0,
$$

and from Lemma 7.4 we have

$$
\left\|w_{1}(t)\right\|_{H_{U}^{1+\sigma}}^{2} \leq Q_{2}\left(\left\|z_{0}\right\|_{H_{U}^{1} \times H_{U}^{1}},\|g\|_{L_{l u}^{2}}\right) e^{2 k_{1} T} \quad \text { for all } t \geq 0,
$$

where $\chi_{[0, T)}(s)$ is the characteristic function of set $[0, T)$.

Remark 8.2. From the proof of Lemma 8.1, we observe that the decomposition $v_{1}(t)$ and $w_{1}(t)$ can also further satisfy that

$$
\left\|v_{1}(t)\right\|_{H_{U}^{1}}^{2} \leq Q_{1}\left(\varrho_{1}\right) \text { for all } t \geq 0 .
$$

In what follows we begin to establish the asymptotic regularity of the solutions. We claim first that

Lemma 8.3. Under Assumption I, there exists a constant $J_{\sigma}$ which depends only on the $H_{U}^{1} \times H_{U}^{1}$-bound of $B_{1}$ such that

$$
\left\|K(t) z_{0}\right\|_{\mathcal{H}_{\sigma}}^{2}=\|w(t)\|_{H_{U}^{1+\sigma}}^{2}+\left\|w_{t}(t)\right\|_{H_{U}^{\sigma}}^{2} \leq J_{\sigma} \quad \text { for all } t \geq 0 \text { and } z_{0} \in B_{1},
$$

where $\sigma=\min \left\{\frac{1}{4}, \frac{N+2-(N-2) \gamma}{2}\right\}$ is given in (6.6).

Proof. Due to (7.6) and the continuous embedding $H_{U}^{1} \hookrightarrow H_{U}^{\sigma}$ for any $0 \leq \sigma \leq 1$, we only need to prove $\|w(t)\|_{H_{U}^{1+\sigma}}^{2} \leq J_{\sigma}$ for some constant $J_{\sigma}$.

Let $\theta$ and $\theta_{y}(x)=\theta(|x-y|)$ as in Lemma 7.4

Multiplying (6.5) by $\theta_{y} A^{\sigma}\left(\theta_{y} w(t)\right)$, we have

$$
\begin{gathered}
\left\langle w_{t t}(t), \theta_{y} A^{\sigma}\left(\theta_{y} w(t)\right)\right\rangle+\left\langle w_{t}(t), \theta_{y} A^{\sigma}\left(\theta_{y} w(t)\right)\right\rangle-\left\langle\Delta w_{t}(t), \theta_{y} A^{\sigma}\left(\theta_{y} w(t)\right)\right\rangle \\
-\left\langle\Delta w, \theta_{y} A^{\sigma}\left(\theta_{y} w(t)\right)\right\rangle+\left\langle f(u)-f_{0}(v), \theta_{y} A^{\sigma}\left(\theta_{y} w(t)\right)\right\rangle \\
=\left\langle g(x)+v(t), \theta_{y} A^{\sigma}\left(\theta_{y} w(t)\right)\right\rangle .
\end{gathered}
$$


Similar to Lemma 7.4, in the following we will deal with each term in (8.4) one by one:

$$
\begin{gathered}
\left\langle w_{t t}, \theta_{y} A^{\sigma}\left(\theta_{y} w(t)\right)\right\rangle=\frac{d}{d t}\left\langle\left(\theta_{y} w\right)_{t}, A^{\sigma}\left(\theta_{y} w(t)\right)\right\rangle-\left\langle\left(\theta_{y} w\right)_{t}, A^{\sigma}\left(\theta_{y} w(t)\right)_{t}\right\rangle, \\
\left|\left\langle w_{t}(t), \theta_{y} A^{\sigma}\left(\theta_{y} w(t)\right)\right\rangle\right| \leq\left\|w_{t}(t)\right\|_{L_{U}^{2}}\left(\int_{\mathbb{R}^{N}}\left|A^{\sigma}\left(\theta_{y} w(t)\right)\right|^{2}\right)^{\frac{1}{2}} \\
\left\langle-\Delta w_{t}(t), \theta_{y} A^{\sigma}\left(\theta_{y} w(t)\right)\right\rangle \\
=\frac{1}{2} \frac{d}{d t} \int_{\mathbb{R}^{N}}\left|A^{\frac{1+\sigma}{2}}\left(\theta_{y} w\right)\right|^{2}+\left\langle 2 \nabla \theta_{y} \cdot \nabla w_{t}+\Delta \theta_{y} w_{t}, A^{\sigma}\left(\theta_{y} w\right)\right\rangle
\end{gathered}
$$

and

$$
\begin{aligned}
& \left\langle-\Delta w(t), \theta_{y} A^{\sigma}\left(\theta_{y} w(t)\right)\right\rangle \\
& \quad=\int_{\mathbb{R}^{N}}\left|A^{\frac{1+\sigma}{2}}\left(\theta_{y} w\right)\right|^{2}+\left\langle 2 \nabla \theta_{y} \cdot \nabla w+\Delta \theta_{y} w, A^{\sigma}\left(\theta_{y} w\right)\right\rangle,
\end{aligned}
$$

where

$$
\begin{aligned}
& \left|\left\langle 2 \nabla \theta_{y} \cdot \nabla w_{t}+\Delta \theta_{y} w_{t}, A^{\sigma}\left(\theta_{y} w\right)\right\rangle\right| \leq C\left\|w_{t}(t)\right\|_{H_{U}^{1}}\left(\int_{\mathbb{R}^{N}}\left|A^{\sigma}\left(\theta_{y} w(t)\right)\right|^{2}\right)^{\frac{1}{2}}, \\
& \left|\left\langle 2 \nabla \theta_{y} \cdot \nabla w+\Delta \theta_{y} w, A^{\sigma}\left(\theta_{y} w\right)\right\rangle\right| \leq C\|w(t)\|_{H_{U}^{1}}\left(\int_{\mathbb{R}^{N}}\left|A^{\sigma}\left(\theta_{y} w(t)\right)\right|^{2}\right)^{\frac{1}{2}} .
\end{aligned}
$$

For the nonlinearity, applying (1.3) and Lemma 8.1, we have

$$
\begin{aligned}
\left|\left\langle f(u)-f(v), \theta_{y} A^{\sigma}\left(\theta_{y} w(t)\right)\right\rangle\right| & \leq C \int_{\mathbb{R}^{N}}\left(1+|u|^{\frac{4}{N-2}}+|v|^{\frac{4}{N-2}}\right) \theta_{y}|w|\left|A^{\sigma}\left(\theta_{y} w\right)\right|, \\
\int_{\mathbb{R}^{N}}|u|^{\frac{4}{N-2}} \theta_{y}|w|\left|A^{\sigma}\left(\theta_{y} w\right)\right| & \leq C \int_{\mathbb{R}^{N}}\left(\left|v_{1}\right|^{\frac{4}{N-2}}+\left|w_{1}\right|^{\frac{4}{N-2}}\right) \theta_{y}|w|\left|A^{\sigma}\left(\theta_{y} w\right)\right| .
\end{aligned}
$$

Obviously,

$$
\int_{\mathbb{R}^{N}} \theta_{y}|w|\left|A^{\sigma}\left(\theta_{y} w\right)\right| \leq C\|w\|_{L_{U}^{2}}\left\|A^{\sigma}\left(\theta_{y} w\right)\right\|_{L^{2}}
$$

and

$$
\begin{aligned}
\int_{\mathbb{R}^{N}} \theta_{y}|w|\left|v_{1}\right| \frac{4}{N-2}\left|A^{\sigma}\left(\theta_{y} w\right)\right| \\
=\int_{B(y, 1)}\left|v_{1}\right|^{\frac{4}{N-2}}\left|\theta_{y} w\right|\left|A^{\sigma}\left(\theta_{y} w\right)\right| \\
\leq C\left(\int_{B(y, 1)}\left|v_{1}\right|^{\frac{2 N}{N-2}}\right)^{\frac{2}{N}}\left(\int_{\mathbb{R}^{N}}\left|\theta_{y} w\right|^{\frac{2 N}{N-2-2 \sigma}}\right)^{\frac{N-2-2 \sigma}{2 N}} \\
\\
\cdot\left(\int_{\mathbb{R}^{N}}\left|A^{\sigma}\left(\theta_{y} w\right)\right| \frac{2 N}{N-2+2 \sigma}\right)^{\frac{N-2+2 \sigma}{2 N}} \\
\leq C\left\|v_{1}\right\|_{H_{U}^{1}}^{\frac{4}{N-2}}\left(\left\|\theta_{y} w\right\|_{L^{2}}+\left\|A^{\frac{1+\sigma}{2}}\left(\theta_{y} w\right)\right\|_{L^{2}}\right)\left(\left\|A^{\sigma}\left(\theta_{y} w\right)\right\|_{L^{2}}+\left\|A^{\frac{1+\sigma}{2}}\left(\theta_{y} w\right)\right\|_{L^{2}}\right) \\
(8.12) \\
\leq C_{M}\left\|v_{1}\right\|_{H_{U}^{1}}^{2} \int_{\mathbb{R}^{N}}\left|A^{\frac{1+\sigma}{2}}\left(\theta_{y} w\right)\right|^{2}+\frac{1}{8} \int_{\mathbb{R}^{N}}\left|A^{\frac{1+\sigma}{2}}\left(\theta_{y} w\right)\right|^{2}+C_{M}\left(1+\left\|\theta_{y} w\right\|_{L^{2}}^{2}\right),
\end{aligned}
$$


where we used Remark 8.2 and where the constant $C_{M}$ depends on the $\mathcal{H}_{0}$-bound of $B_{1}$.

Moreover, using (8.2) we have

$$
\begin{aligned}
& \int_{\mathbb{R}^{N}} \theta_{y}|w|\left|w_{1}\right|^{\frac{4}{N-2}}\left|A^{\sigma}\left(\theta_{y} w\right)\right| \\
& \quad \leq C\left(\int_{B(y, 1)}\left|w_{1}\right|^{\frac{2 N}{N-2-2 \sigma}}\right)^{\frac{2(N-2-2 \sigma)}{N(N-2)}}\left\|\theta_{y} w\right\|_{L^{p}}\left(\int_{\mathbb{R}^{N}}\left|A^{\sigma}\left(\theta_{y} w\right)\right|^{\frac{2 N}{N-2+2 \sigma}}\right)^{\frac{N-2+2 \sigma}{2 N}} \\
& \quad \leq C\left\|w_{1}\right\|_{H_{U}^{1+\sigma}}^{\frac{4}{N-2}}\left\|\theta_{y} w\right\|_{L^{p}}\left(\left\|A^{\sigma}\left(\theta_{y} w\right)\right\|_{L^{2}}+\left\|A^{\frac{1+\sigma}{2}}\left(\theta_{y} w\right)\right\|_{L^{2}}\right)
\end{aligned}
$$

$$
\leq \frac{1}{8} \int_{\mathbb{R}^{N}}\left|A^{\frac{1+\sigma}{2}}\left(\theta_{y} w\right)\right|^{2}+C K_{\varepsilon}\left(1+\left\|\theta_{y} w\right\|_{L^{2}}^{2}\right),
$$

where $p=\frac{2 N(N-2)}{(N-2)^{2}-2(N-6) \sigma}<\frac{2 N}{N-2-2 \sigma}$, and we also used the interpolation inequality.

On the other hand, we have

$$
\begin{aligned}
& \int_{\mathbb{R}^{N}} \theta_{y}|w||v|^{\frac{4}{N-2}}\left|A^{\sigma}\left(\theta_{y} w\right)\right| \\
& \leq C\left(\int_{B(y, 1)}|v|^{\frac{2 N}{N-2}}\right)^{\frac{2}{N}}\left(\int_{\mathbb{R}^{N}}\left|\theta_{y} w\right|^{\frac{2 N}{N-2-2 \sigma}}\right) \frac{N-2-2 \sigma}{2 N}\left(\int_{\mathbb{R}^{N}}\left|A^{\sigma}\left(\theta_{y} w\right)\right|^{\frac{2 N}{N-2+2 \sigma}}\right)^{\frac{N-2+2 \sigma}{2 N}} \\
& \leq C\|v\|_{H_{U}^{1}}^{\frac{4}{N-2}}\left(\left\|\theta_{y} w\right\|_{L^{2}}+\left\|A^{\frac{1+\sigma}{2}}\left(\theta_{y} w\right)\right\|_{L^{2}}\right)\left(\left\|A^{\sigma}\left(\theta_{y} w\right)\right\|_{L^{2}}+\left\|A^{\frac{1+\sigma}{2}}\left(\theta_{y} w\right)\right\|_{L^{2}}\right)
\end{aligned}
$$

$$
\leq C_{M}\|v\|_{H_{U}^{1}}^{\frac{4}{N-2}} \int_{\mathbb{R}^{N}}\left|A^{\frac{1+\sigma}{2}}\left(\theta_{y} w\right)\right|^{2}+C_{M}\left(1+\left\|\theta_{y} w\right\|_{L^{2}}^{2}\right),
$$

and thanks to Lemmas 7.1 and 7.3 , we can take $T_{1}$ large enough such that

$$
\|v(t)\|_{H_{U}^{1}}^{\frac{4}{N-2}} \leq \frac{1}{8 C_{M}} \text { for all } t \geq T_{1} .
$$

Finally, for the subcritical term, we have

$$
\begin{aligned}
\left|\left\langle f_{1}(v), \theta_{y} A^{\sigma}\left(\theta_{y} w\right)\right\rangle\right| & \leq C \int_{\mathbb{R}^{N}}\left(1+|v|^{\gamma}\right) \theta_{y}\left|A^{\sigma}\left(\theta_{y} w\right)\right| \\
& \leq C\left(1+\int_{B(y, 1)} \theta_{y}|v|^{\gamma} \frac{2 N}{N+2-2 \sigma}\right)^{\frac{N+2-2 \sigma}{2 N}}\left\|A^{\sigma}\left(\theta_{y} w\right)\right\|_{L^{\frac{2 N}{N-2+2 \sigma}}} \\
& \leq C\left(1+\|v\|_{H_{U}^{1}}^{\gamma}\right)\left(\left\|A^{\sigma}\left(\theta_{y} w\right)\right\|_{L^{2}}+\left\|A^{\frac{1+\sigma}{2}}\left(\theta_{y} w\right)\right\|_{L^{2}}\right) \\
& \leq C_{M}\left(1+\left\|\theta_{y} w\right\|_{L^{2}}^{2}\right)+\frac{1}{8} \int_{\mathbb{R}^{N}}\left|A^{\frac{1+\sigma}{2}}\left(\theta_{y} w\right)\right|^{2},
\end{aligned}
$$

and for the forcing term, we have

$$
\left|\left\langle g+v, \theta_{y} A^{\sigma}\left(\theta_{y} w\right)\right\rangle\right| \leq C\left(\|g\|_{L_{U}^{2}}+\|v\|_{L_{U}^{2}}\right)\left(\int_{\mathbb{R}^{N}}\left|A^{\sigma}\left(\theta_{y} w\right)\right|^{2}\right)^{\frac{1}{2}}
$$


Hence, summarizing the above estimates into (8.4), and due to (7.6), we have: for all $t \geq T_{1}$,

$$
\begin{array}{r}
\frac{d}{d t} \int_{\mathbb{R}^{N}}\left(\left|A^{\frac{1+\sigma}{2}}\left(\theta_{y} w(t)\right)\right|^{2}+2\left(\theta_{y} w\right)_{t} \cdot A^{\sigma}\left(\theta_{y} w\right)\right)+\int_{\mathbb{R}^{N}}\left|A^{\frac{1+\sigma}{2}}\left(\theta_{y} w(t)\right)\right|^{2} \\
\leq C_{M}\left\|v_{1}(t)\right\|_{H_{U}^{1}}^{2} \int_{\mathbb{R}^{N}}\left|A^{\frac{1+\sigma}{2}}\left(\theta_{y} w(t)\right)\right|^{2}+C_{M, K_{\varepsilon},\|g\|_{L_{l u}^{2}}}
\end{array}
$$

then, noting (18.6), (7.6) and Remark 8.2 again, we get finally that

$$
\frac{d}{d t} E_{2}(t)+\left(1-C_{M}\left\|v_{1}(t)\right\|_{H_{U}^{1}}^{2}\right) E_{2}(t) \leq C^{\prime}
$$

where $E_{2}(t)=\int_{\mathbb{R}^{N}}\left(\left|A^{\frac{1+\sigma}{2}}\left(\theta_{y} w(t)\right)\right|^{2}+2\left(\theta_{y} w(t)\right)_{t} \cdot A^{\sigma}\left(\theta_{y} w(t)\right)\right)$, and the positive constant $C^{\prime}$ depends on the $H_{U}^{1} \times H_{U}^{1}$-bound of $B_{1}, K_{\varepsilon}$ and $\|g\|_{L_{l u}^{2}}$.

Therefore, applying the Gronwall inequality and integrating over $\left[1+T_{1}, t\right]$, we obtain that

$$
E_{2}(t) \leq e^{-\int_{T_{1}+1}^{t}\left(1-C_{M}\left\|v_{1}(s)\right\|_{H_{U}^{1}}^{2}\right) d s} E_{2}\left(1+T_{1}\right)+C^{\prime} \int_{T_{1}+1}^{t} e^{\int_{t}^{s}\left(1-C_{M}\left\|v_{1}(\tau)\right\|_{H_{U}^{1}}^{2}\right) d \tau} d s .
$$

Taking $\varepsilon$ (in (8.3) ) small enough (for example) such that $\varepsilon<\frac{1}{2 C_{M}}$, we have

$$
\begin{aligned}
\int_{T_{1}+1}^{t} e^{\int_{t}^{s}\left(1-C_{M}\left\|v_{1}(\tau)\right\|_{H_{U}^{1}}^{2}\right) d \tau} d s & \leq e^{C_{M} C_{\varepsilon}} \int_{T_{1}+1}^{t} e^{\left(1-C_{M} \varepsilon\right)(s-t)} d s \\
& \leq e^{C_{M} C_{\varepsilon}} \int_{T_{1}+1}^{t} e^{(s-t) / 2} d s \leq 2 e^{C_{M} C_{\varepsilon}}
\end{aligned}
$$

and

$$
e^{-\int_{T_{1}+1}^{t}\left(1-C_{M}\left\|v_{1}(s)\right\|_{H_{U}^{1}}^{2}\right) d s} \leq e^{-\frac{1}{2}\left(t-T_{1}-1\right)} e^{C_{M} C_{\varepsilon}} .
$$

Substituting (8.19)-(8.20) into (8.18) and using (7.6) again, we can get that, for all $t \geq 1+T_{1}$,

$$
\int_{\mathbb{R}^{N}}\left|A^{\frac{1+\sigma}{2}}\left(\theta_{y} w(t)\right)\right|^{2} \leq e^{-\frac{1}{2}\left(t-T_{1}-1\right)} e^{C_{M} C_{\varepsilon}} \int_{\mathbb{R}^{N}}\left|A^{\frac{1+\sigma}{2}}\left(\theta_{y} w\left(1+T_{1}\right)\right)\right|^{2}+C^{\prime} e^{C_{M} C_{\varepsilon}} .
$$

Hence, noting again that Lemma 7.4 and $T_{1}$ is fixed, the proof is completed.

Lemma 8.4. Assume $B_{\sigma} \subset B_{1}$ and $B_{\sigma}$ is bounded in $\mathcal{H}_{\sigma}$. Then there exists a constant $M_{\sigma}(>0)$ which depends only on the $\mathcal{H}_{\sigma}$-bound of $B_{\sigma}$ such that

$$
\left\|S(t) B_{\sigma}\right\|_{\mathcal{H}_{\sigma}} \leq M_{\sigma} \quad \text { for all } t \geq 0 .
$$

Proof. Multiplying (1.1) by $\theta_{y} A^{\sigma}\left(\theta_{y} u_{t}(t)+\theta_{y} u(t)\right)$, we have

$$
\begin{gathered}
\frac{1}{2} \frac{d}{d t} \int_{\mathbb{R}^{N}}\left|A^{\frac{\sigma}{2}}\left(\theta_{y} u_{t}(t)+\theta_{y} u(t)\right)\right|^{2}-\left\langle\Delta u_{t}, \theta_{y} A^{\sigma}\left(\theta_{y} u_{t}(t)+\theta_{y} u(t)\right)\right\rangle \\
\quad-\left\langle\Delta u, \theta_{y} A^{\sigma}\left(\theta_{y} u_{t}(t)+\theta_{y} u(t)\right)\right\rangle+\left\langle f(u), \theta_{y} A^{\sigma}\left(\theta_{y} u_{t}(t)+\theta_{y} u(t)\right)\right\rangle \\
=\left\langle g, \theta_{y} A^{\sigma}\left(\theta_{y} u_{t}(t)+\theta_{y} u(t)\right)\right\rangle .
\end{gathered}
$$

Here we only deal with the nonlinear term; we can deal with the other terms by similar calculations used in previous lemmas. 
We will use Lemma 8.1, at first,

$$
\left\langle f(u), \theta_{y} A^{\sigma}\left(\theta_{y} u_{t}(t)+\theta_{y} u(t)\right)\right\rangle \leq C \int_{\mathbb{R}^{N}} \theta_{y}\left(1+|u|^{\frac{N+2}{N-2}}\right)\left|A^{\sigma}\left(\theta_{y} u_{t}(t)+\theta_{y} u(t)\right)\right|,
$$

and from (8.1) we have

$$
\begin{aligned}
\int_{\mathbb{R}^{N}} \theta_{y}|u|^{\frac{N+2}{N-2}}\left|A^{\sigma}\left(\theta_{y} u_{t}\right)\right| & =\int_{\mathbb{R}^{N}} \theta_{y}|u|^{\frac{4}{N-2}}|u|\left|A^{\sigma}\left(\theta_{y} u_{t}\right)\right| \\
& \leq \int_{\mathbb{R}^{N}} \theta_{y}\left|v_{1}+w_{1}\right|^{\frac{4}{N-2}}|u|\left|A^{\sigma}\left(\theta_{y} u_{t}\right)\right| \\
& \leq C \int_{\mathbb{R}^{N}} \theta_{y}\left(\left|v_{1}\right|^{\frac{4}{N-2}}+\left|w_{1}\right|^{\frac{4}{N-2}}\right)|u|\left|A^{\sigma}\left(\theta_{y} u_{t}\right)\right|
\end{aligned}
$$

Here,

$$
\begin{aligned}
& \int_{\mathbb{R}^{N}} \theta_{y}\left|v_{1}\right|^{\frac{4}{N-2}}\left|u \| A^{\sigma}\left(\theta_{y} u_{t}\right)\right| \\
& \leq\left\|v_{1}\right\|_{H_{U}^{1}}^{\frac{4}{N-2}}\left\|\theta_{y} u\right\|_{L^{\frac{2 N}{N-2-2 \sigma}}}\left\|A^{\sigma}\left(\theta_{y} u_{t}\right)\right\|_{L^{\frac{2 N}{N-2+2 \sigma}}} \\
& \leq C\left\|v_{1}\right\|_{H_{U}^{1}}^{\frac{4}{N-2}}\left(\left\|\theta_{y} u\right\|_{L^{2}}+\left\|A^{\frac{1+\sigma}{2}}\left(\theta_{y} u\right)\right\|_{L^{2}}\right)\left(\left\|\theta_{y} u_{t}\right\|_{L^{2}}+\left\|A^{\frac{1+\sigma}{2}}\left(\theta_{y} u_{t}\right)\right\|_{L^{2}}\right) \\
& \leq C_{M} C_{\epsilon}\left\|v_{1}\right\|_{H_{U}^{1}}^{\frac{4}{N-2}}\left(1+\left\|A^{\frac{1+\sigma}{2}}\left(\theta_{y} u\right)\right\|_{L^{2}}^{2}\right)+\epsilon\left\|A^{\frac{1+\sigma}{2}}\left(\theta_{y} u_{t}\right)\right\|_{L^{2}}^{2}
\end{aligned}
$$

$$
\leq C_{M} C_{\epsilon}\left\|v_{1}\right\|_{H_{U}^{1}}^{2}\left(1+\left\|A^{\frac{1+\sigma}{2}}\left(\theta_{y} u\right)\right\|_{L^{2}}^{2}\right)+\epsilon\left(1+\left\|A^{\frac{1+\sigma}{2}}\left(\theta_{y} u\right)\right\|_{L^{2}}^{2}+\left\|A^{\frac{1+\sigma}{2}}\left(\theta_{y} u_{t}\right)\right\|_{L^{2}}^{2}\right)
$$

where $C_{M}$ is a constant which depends on the $\mathcal{H}_{0}$-bound of initial data (see Remark 8.2). Similar to (8.13), we have

$$
\begin{aligned}
& \int_{\mathbb{R}^{N}} \theta_{y}\left|w_{1}\right|^{\frac{4}{N-2}}\left|u \| A^{\sigma}\left(\theta_{y} u_{t}\right)\right| \\
& \leq C\left\|w_{1}\right\|_{H_{U}^{1+\sigma}}^{\frac{4}{N-2}}\left\|\theta_{y} u\right\|_{L^{p}}\left(\left\|A^{\sigma}\left(\theta_{y} u_{t}\right)\right\|_{L^{2}}+\left\|A^{\frac{1+\sigma}{2}}\left(\theta_{y} u_{t}\right)\right\|_{L^{2}}\right)
\end{aligned}
$$

$$
\leq \frac{1}{8}\left(\int_{\mathbb{R}^{N}}\left|A^{\frac{1+\sigma}{2}}\left(\theta_{y} u\right)\right|^{2}+\int_{\mathbb{R}^{N}}\left|A^{\frac{1+\sigma}{2}}\left(\theta_{y} u_{t}\right)\right|^{2}\right)+C K_{\varepsilon}\left(1+\left\|\theta_{y} u\right\|_{L^{2}}^{2}+\left\|\theta_{y} u_{t}\right\|_{L^{2}}^{2}\right),
$$

where $p=\frac{2 N(N-2)}{(N-2)^{2}-2(N-6) \sigma}<\frac{2 N}{N-2-2 \sigma}$ and where we used interpolation inequality and (8.2).

Moreover,

$$
\begin{aligned}
\int_{\mathbb{R}^{N}} \theta_{y}|u|^{\frac{N+2}{N-2}}\left|A^{\sigma}\left(\theta_{y} u\right)\right| \leq C_{M} C_{\epsilon}\left\|v_{1}\right\|_{H_{U}^{1}}^{2}\left(1+\left\|A^{\frac{1+\sigma}{2}}\left(\theta_{y} u\right)\right\|_{L^{2}}^{2}\right) & \\
& +\epsilon\left(1+\left\|A^{\frac{1+\sigma}{2}}\left(\theta_{y} u\right)\right\|_{L^{2}}^{2}\right)+C K_{\varepsilon}\left(1+\left\|\theta_{y} u\right\|_{L^{2}}^{2}\right) .
\end{aligned}
$$


Therefore, by taking $\epsilon$ small enough and noticing (7.2), we have

$$
\begin{aligned}
& \frac{d}{d t}\left(\left\|A^{\frac{\sigma}{2}}\left(\theta_{y} u_{t}(t)+\theta_{y} u(t)\right)\right\|_{L^{2}}^{2}+2\left\|A^{\frac{1+\sigma}{2}}\left(\theta_{y} u(t)\right)\right\|_{L^{2}}^{2}\right) \\
& +\left(\frac{1}{2}-C_{M} C_{\epsilon}\left\|v_{1}(t)\right\|_{H_{U}^{1}}^{2}\right)\left(\left\|A^{\frac{\sigma}{2}}\left(\theta_{y} u_{t}(t)+\theta_{y} u(t)\right)\right\|_{L^{2}}^{2}+2\left\|A^{\frac{1+\sigma}{2}}\left(\theta_{y} u(t)\right)\right\|_{L^{2}}^{2}\right) \\
& \leq C_{M, K_{\varepsilon},\|g\|_{L_{l u}^{2}}, \epsilon} .
\end{aligned}
$$

Using the Gronwall inequality and integrating over $[0, t]$, we get that

$$
\begin{aligned}
& \left\|A^{\frac{\sigma}{2}}\left(\theta_{y} u_{t}(t)+\theta_{y} u(t)\right)\right\|_{L^{2}}^{2}+2\left\|A^{\frac{1+\sigma}{2}}\left(\theta_{y} u(t)\right)\right\|_{L^{2}}^{2} \\
& \leq e^{-\int_{0}^{t}\left(\frac{1}{2}-C_{M} C_{\epsilon}\left\|v_{1}(s)\right\|_{H_{U}^{1}}^{2}\right) d s}\left(\left\|A^{\frac{\sigma}{2}}\left(\theta_{y} u_{t}(0)+\theta_{y} u(0)\right)\right\|_{L^{2}}^{2}+2\left\|A^{\frac{1+\sigma}{2}}\left(\theta_{y} u(0)\right)\right\|_{L^{2}}^{2}\right)
\end{aligned}
$$

$$
+C_{M, K_{\varepsilon},\|g\|_{L_{l u}^{2}}, \epsilon} \int_{0}^{t} e^{\int_{t}^{s}\left(\frac{1}{2}-C_{M} C_{\epsilon}\left\|v_{1}(\tau)\right\|_{H_{U}^{1}}^{2}\right) d \tau} d s .
$$

Then, we can complete the proof by the same calculation as that for (8.21), and we only need to note that $\left(u_{0}, v_{0}\right)$ now also belongs to $\mathcal{H}_{\sigma}$.

In the following, based on Lemmas 8.3 and 8.4 above, we can perform the bootstrap arguments; their proofs are similar to those for Lemmas 8.3 and 8.4 (e.g., see [27, 29, 30, for the bounded domain case). We also remark that Lemma 8.4 makes $f$ become subcritical to some extent; consequently, the proofs of Lemmas 8.5 and 8.6 below are simpler than Lemmas 8.3 and 8.4 .

Lemma 8.5. For each $\sigma \leq \kappa \leq 1$, assume $B_{\kappa} \subset B_{1}$ and $B_{\kappa}$ is bounded in $\mathcal{H}_{\kappa}$. Then there exists a constant $M_{\kappa}$ which depends only on the $\mathcal{H}_{\kappa}$-bound of $B_{\kappa}$ such that

$$
\left\|S(t) B_{\kappa}\right\|_{\mathcal{H}_{\kappa}} \leq M_{\kappa} \quad \text { for all } t \geq 0 .
$$

Lemma 8.6. For each $\kappa \in\left[\sigma, 1-\min \left\{\sigma, \frac{4 \sigma}{N-2}\right\}\right]$, if the initial data set $B_{\kappa} \subset B_{1}$ and $B_{\kappa}$ is bounded in $\mathcal{H}_{\kappa}$, then the decomposed ingredient $\left(w(t), w_{t}(t)\right)$ (the solutions of (6.5) satisfies

$$
\left\|K(t) B_{\kappa}\right\|_{\mathcal{H}_{\kappa+s}} \leq J_{\kappa} \quad \text { for all } t \geq 0,
$$

where the constant $J_{\kappa}$ depends only on the $\mathcal{H}_{\kappa}$-bound of $B_{\kappa}$ and $s=\min \left\{\sigma, \frac{4 \sigma}{N-2}\right\}$.

\section{Proofs of the main Results}

9.1. Proof of Theorem 1.1. In this subsection, we mainly verify Theorem 1.1,

We first recall the following attraction transitivity lemma:

Lemma 9.1 ([17]). Let $K_{1}, K_{2}, K_{3}$ be subsets of $\mathcal{H}$ such that

$$
\operatorname{dist}_{\mathcal{H}}\left(S(t) K_{1}, K_{2}\right) \leq L_{1} e^{-\nu_{1} t}, \quad \operatorname{dist}_{\mathcal{H}}\left(S(t) K_{2}, K_{3}\right) \leq L_{2} e^{-\nu_{2} t},
$$

for some $\nu_{1}, \nu_{2}>0$ and $L_{1}, L_{2}>0$. Assume also that for all $z_{1}, z_{2} \in \bigcup_{t \geq 0} S(t) K_{j}$ $(j=1,2,3)$ there holds

$$
\left\|S(t) z_{1}-S(t) z_{2}\right\| \leq L_{0} e^{\nu_{0} t}\left\|z_{1}-z_{2}\right\|
$$

for some $\nu_{0}>0$ and some $L_{0} \geq 0$. Then it follows that

$$
\operatorname{dist}_{\mathcal{H}}\left(S(t) K_{1}, K_{3}\right) \leq L e^{-\nu t},
$$

where $\nu=\frac{\nu_{1} \nu_{2}}{\nu_{0}+\nu_{1}+\nu_{2}}$ and $L=L_{0} L_{1}+L_{2}$. 
Based on the above preliminary lemmas, we are now in the position to prove Theorem 1.1.

Proof of Theorem 1.1. For the set $B_{1}$ given in (7.1), from Lemma 8.3 and Lemma 7.3 we know that there is a set $A_{\sigma}$ which is bounded in $\mathcal{H}_{\sigma}$ such that

$$
\operatorname{dist}_{\mathcal{H}_{0}}\left(S(t) B_{1}, A_{\sigma}\right) \leq \operatorname{dist}_{\mathcal{H}_{0}}\left(D(t) B_{1}, A_{\sigma}\right) \leq Q_{1}\left(\left\|B_{1}\right\|_{\mathcal{H}_{0}}\right) e^{-k_{0} t} .
$$

Applying Lemma 8.6 and Lemma 7.3 to $A_{\sigma}$, we see that there is a set $A_{\sigma+s}$ (given in Lemma 8.6) which is bounded in $\mathcal{H}_{\sigma+s}$, such that

$$
\operatorname{dist}_{\mathcal{H}_{0}}\left(S(t) A_{\sigma}, A_{\sigma+s}\right) \leq \operatorname{dist}_{\mathcal{H}_{0}}\left(D(t) A_{\sigma}, A_{\sigma+s}\right) \leq Q_{1}\left(\left\|A_{\sigma}\right\|_{\mathcal{H}_{0}}\right) e^{-k_{0}^{\prime} t},
$$

where $k_{0}^{\prime}$ depends on the $\mathcal{H}_{0}$-bound of $A_{\sigma}$.

Combining this with Remark 5.6 we know that the conditions in Lemma 9.1 are all satisfied. Hence, we have

$$
\operatorname{dist}_{\mathcal{H}_{0}}\left(S(t) B_{1}, A_{\sigma+s}\right) \leq C Q_{1}\left(\left\|B_{1}\right\|_{\mathcal{H}_{0}}\right) e^{-k_{0}^{\prime \prime} t}
$$

for two appropriate constants $C$ and $k_{0}^{\prime \prime}$.

Since $\sigma=\min \left\{\frac{1}{4}, \frac{N+2-(N-2) \gamma}{2}\right\}$ and $s=\min \left\{\sigma, \frac{4 \sigma}{N-2}\right\}$ are fixed, by finite steps (e.g., at most by $\left[\frac{1}{s}\right]+2$ steps) we can infer that there is a bounded (in $\mathcal{H}_{1}$ ) set $\mathcal{B}_{1} \subset \mathcal{H}_{1}$ such that

$$
\operatorname{dist}_{\mathcal{H}_{0}}\left(S(t) B_{1}, \mathcal{B}_{1}\right) \leq Q\left(\left\|B_{1}\right\|_{\mathcal{H}_{0}}\right) e^{-\nu t}
$$

Note further that all the constants in (9.1) depend only on $\left\|B_{1}\right\|_{\mathcal{H}_{0}}$.

Now, for any bounded (in $\mathcal{H}_{0}$ ) set $B$, from Theorem 4.1 and Lemma 4.2 we see that there is a $T_{B}$ such that

$$
S(t) B \subset B_{1} \quad \text { for all } t \geq T_{B} .
$$

Hence,

$$
\operatorname{dist}_{\mathcal{H}_{0}}\left(S(t) B, B_{1}\right) \leq M e^{\nu T_{B}} e^{-\nu t},
$$

where $M=\sup \left\{\|S(t) B\|_{\mathcal{H}_{0}}, 0 \leq t \leq T_{B}\right\}<\infty$.

Finally, we apply the attraction transitivity lemma, i.e., Lemma 9.1, again to (9.1) and (9.2), and this completes the proof of Theorem 1.1.

9.2. Proof of Theorem 1.2. In this subsection, we will prove one of our main results, Theorem 1.2. From Theorem 1.1 we know that $\{S(t)\}_{t \geq 0}$ has a bounded $\left(\mathcal{H}_{0}, H_{l u}^{1} \times H_{l u}^{1}\right)$-attracting set $\mathcal{B}_{1}$ (given in Theorem 1.1); then we only need to verify the corresponding asymptotic compactness and continuity.

We first deduce some continuity of $S(t)$ with respect to the $H_{\rho}^{1} \times H_{\rho}^{1}$-norm in the bounded absorbing set $B_{1}$ (given in (7.1)).

Lemma 9.2 (Continuity w.r.t. the $H_{\rho}^{1} \times H_{\rho}^{1}$-norm). Let $z_{0}^{n}=\left(u_{0}^{n}, v_{0}^{n}\right) \in B_{1}$, $n=1,2, \cdots$, be a convergent sequence with respect to the $H_{\rho}^{1} \times L_{\rho}^{2}$-norm. Then for any $t \geq 0, S(t) z_{0}^{n}$ is also a convergent sequence with respect to the $H_{\rho}^{1} \times H_{\rho}^{1}$-norm.

Proof. Let $\left(u^{i}(t), u_{t}^{i}(t)\right)(i=1,2)$ be the corresponding solution to $\left(u_{0}^{i}, v_{0}^{i}\right) \in B_{1}$, and let $z(t)=u^{1}(t)-u^{2}(t)$. Then $z$ satisfies

$$
z_{t t}+z_{t}-\Delta z_{t}-\Delta z+f\left(u^{1}(t)\right)-f\left(u^{2}(t)\right)=0,
$$

with the initial condition

$$
\left(z(0), z_{t}(0)\right)=\left(u_{0}^{1}, v_{0}^{1}\right)-\left(u_{0}^{2}, v_{0}^{2}\right) .
$$


Multiplying (9.3) by $\rho z_{t}$, and by some calculations as before, we have

$$
\int_{\mathbb{R}^{N}} \rho\left(\left|z_{t}(t)\right|^{2}+\left|\nabla z_{t}(t)\right|^{2}\right) \leq C_{M} \int_{\mathbb{R}^{N}} \rho\left(\left|z_{t}(t)\right|+|\nabla z(t)|^{2}+|z(t)|^{2}\right),
$$

where $C_{M}$ only depends on the $H_{l u}^{1} \times L_{l u}^{2}$-bound of $B_{1}$. This, combined with the continuity of $S(t)$ with respect to the $H_{\rho}^{1} \times L_{\rho}^{2}$-norm and the arbitrariness of $\left(u_{0}^{i}, v_{0}^{i}\right)$, implies that $S(t)$ is continuous in $B_{1}$ with respect to the $H_{\rho}^{1} \times H_{\rho}^{1}$-norm.

Proof of Theorem 1.2, We only need to verify the necessary $\left(\mathcal{H}_{0}, H_{\rho}^{1} \times H_{\rho}^{1}\right)$-asymptotic compactness. This is a direct corollary of the compactness of $B_{1}$ w.r.t. the $H_{\rho}^{1} \times L_{\rho}^{2}$-norm (by compact embedding) and the continuity (9.4).

\section{ACKNOWLEDGMENTS}

The authors would like to thank the referee for many helpful comments and suggestions, which essentially improved this paper.

\section{REFERENCES}

1. J. Arrieta, A.N. Carvalho and J.K. Hale, A damped hyperbolic equation with critical exponent, Comm. Partial Differential Equations, 17 (1992), 841-866. MR.1177295 (93f:35145)

2. J. Arrieta, J.W. Cholewa, T. Dlotko and A. Rodriguez-Bernal, Linear parabolic equations in locally uniform spaces, Math. Models Methods Appl. Sci., 14 (2004), 253-293. MR2040897 (2004m:35114)

3. A.V. Babin and M.I. Vishik, Attractors of Evolution Equations, North-Holland, Amsterdam, 1992. MR 1156492 (93d:58090)

4. A.V. Babin and M.I. Vishik, Attractors of partial differential evolution equations in an unbounded domain, Proc. Roy. Soc. Edinburgh Sect. A, 116 (1990), 221-243. MR.1084733 (91m:35106)

5. V. Belleri and V. Pata, Attractors for semilinear strongly damped wave equations on $\mathbb{R}^{3}$, Discrete Contin. Dyn. Syst., 7 (2001), 719-735. MR1849655 (2003f:35026)

6. A.N. Carvalho and J.W. Cholewa, Attractors for strongly damped wave equations with critical nonlinearities, Pacific J. Math., 207 (2002), 287-310. MR1972247 (2004b:35023)

7. A.N. Carvalho and J.W. Cholewa, Local well posedness for strongly damped wave equations with critical nonlinearities, Bull. Austral. Math. Soc., 66 (2002), 443-463. MR1939206 (2004b:35228)

8. A.N. Carvalho and T. Dlotko, Partly dissipative systems in uniformly local spaces, Colloq. Math., 100 (2004), 221-242. MR.2107518 (2005i:35113)

9. J.W. Cholewa and T. Dlotko, Global Attractors in Abstract Parabolic Problems, Cambridge University Press, 2000. MR.1778284 (2002f:37132)

10. J.W. Cholewa and T. Dlotko, Hyperbolic equations in uniform spaces, Bulletin of The Polish Academy of Sciences Mathematics, 52 (2004), 249-263. MR2127062 (2006b:35225)

11. J.W. Cholewa and T. Dlotko, Cauchy problems in weighted Lebesgue spaces, Czechoslovak Mathematical Journal, 54 (2004), 991-1013. MR2099352 (2005g:35148)

12. J.W. Cholewa and T. Dlotko, Strongly damped wave equation in uniform spaces, Nonlinear Anal. TMA, 64 (2006), 174-187. MR2183836 (2006f:35183)

13. M. Conti, V. Pata and M. Squassina, Strongly damped wave equations on $\mathbb{R}^{3}$ with critical nonlinearities, Communications in Applied Analysis, 9 (2005), 161-176. MR 2168756 (2006d:35165)

14. M.A. Efendiev, A. Miranville and S.V. Zelik, Infinite-dimensional exponential attractors for nonlinear reaction-diffusion systems in unbounded domains and their approximation, Proc. R. Soc. Lond. A, 460 (2004), 1107-1129. MR2133858 (2005m:37190)

15. M.A. Efendiev and S.V. Zelik, The attractor for a nonlinear reaction-diffusion system in an unbounded domain, Comm. Pure Appl. Math., 54 (2001), 625-688. MR.1815444|(2001m:35035)

16. E. Feireisl, Bounded, locally compact global attractors for semilinear damped wave equations in $\mathbb{R}^{N}$, Differential Integral Equations, 9 (1996), 1147-1156. MR1392099 (97f:35138) 
17. P. Fabrie, C. Galushinski, A. Miranville and S. Zelik, Uniform exponential attractors for a singular perturbed damped wave equation, Discrete Contin. Dyn. Syst., 10 (2004), 211-238. MR 2026192 (2006c:37088)

18. E. Feireisl, Ph. Laurencot, F. Simondon and H. Toure, Compact attractors for reaction diffusion equations in $\mathbb{R}^{N}$, C. R. Acad. Sci. Paris. Sér. I. Math., 319 (1994), 147-151. MR.1288394 (95f:35110)

19. E. Feireisl, Ph. Laurencot and F. Simondon, Global attractors for degenerate parabolic equations on unbounded domains, J. Diff. Equations, 129 (1996), 239-261. MR1404383 (97j:35082)

20. C. Gatti, A. Miranville, V. Pata and S.V. Zelik, Attractors for semilinear equations of viscoelasticity with very low dissipation, R. Mountain J. Math., 38 (2008), 1117-1138.

21. J.K. Hale, Asymptotic Behavior of Dissipative Systems, AMS, Providence, RI, 1988. MR 941371 (89g:58059)

22. N. Karachalios and N. Stavrakakis, Existence of a global attractor for semilinear dissipative wave equations on $\mathbb{R}^{N}$, J. Diff. Equations, 157 (1999), 183-205. MR.1710020 (2000e:35151)

23. T. Kato, The Cauchy problem for quasi-linear symmetric hyperbolic systems, Arch. Rat. Mech. Anal., 58 (1975), 181-205. MR0390516 (52:11341)

24. O.A. Ladyzhenskaya, Attractors for semigroups and evolution equations, Leizioni Lincei, Cambridge Univ. Press, Cambridge, New York, 1991. MR.1133627 (92k:58040)

25. A. Mielke, The complex Ginzburg-Landau equation on large and unbounded domains: sharper bounds and attractors, Nonlinearity, 10 (1997), 199-222. MR1430749 (97m:35242)

26. A. Mielke and G. Schneider, Attractors for modulation equations on unbounded domainsexistence and comparison, Nonlinearity, 8 (1995) 743-768. MR.1355041 (97e:58207)

27. V. Pata and M. Squassina, On the strongly damped wave equation, Comm. Math. Phys., 253 (2005), 511-533. MR2116726 (2005k:35291)

28. V. Pata and S.V. Zelik, Smooth attractors for strongly damped wave equations, Nonlinearity, 19 (2006), 1495-1506. MR2229785 (2007f:35257)

29. C. Sun, D. Cao and J. Duan, Non-autonomous wave dynamics with memory - Asymptotic regularity and uniform attractor, Discrete Contin. Dyn. Syst., Ser. B, 9 (2008), 743-761. MR 2379435

30. C. Sun and M. Yang, Attractors of strongly damped wave equations: asymptotic regularity and exponential attraction, submitted.

31. C. Sun, M. Yang and C. Zhong, Global attractors for hyperbolic equations with critical exponent in locally uniform spaces, submitted.

32. R. Temam, Infinite-Dimensional Dynamical Systems in Mechanics and Physics, Springer, New York, 1997. MR1441312 (98b:58056)

33. M. Yang and C. Sun, Dynamics of strongly damped wave equations in locally uniform spaces II: Infinite-dimensional exponential attractors and their approximation, preparation.

34. S.V. Zelik, The attractor for a nonlinear hyperbolic equation in the unbounded domain, Discrete Contin. Dyn. Syst., 7 (2001), 593-641. MR.1815770 (2001m:35230)

35. S.V. Zelik, Asymptotic regularity of solutions of a nonautonomous damped wave equation with a critical growth exponent, Comm. Pure Appl. Anal., 3 (2004), 921-934. MR2106304 (2005h:35249)

Department of Mathematics, Nanjing University, Nanjing, 210093, People's Republic of China - and - Department of Mathematics, Huazhong University of Science and Technology, Wuhan, 430074, People's Republic of China

E-mail address: yangmeih@gmail.com

Department of Mathematics, Lanzhou University, Lanzhou, 730000, People's Republic of China - And - Institute of Applied Mathematics, Chinese Academy of Sciences, Beijing, 100080, People's Republic of China

E-mail address: cysun@amss.ac.cn

E-mail address: sunchunyou@gmail.com 